\title{
Extending the Clapper-Yule model to rough printing supports
}

\author{
Mathieu Hébert and Roger David Hersch \\ Ecole Polytechnique Fédérale de Lausanne (EPFL), School of Computer and Communication Sciences, \\ Lausanne 1015, Switzerland
}

Received October 13, 2004; revised manuscript received January 28, 2005; accepted February 9, 2005

\begin{abstract}
The Clapper-Yule model is the only classical spectral reflection model for halftone prints that takes explicitly into account both the multiple internal reflections between the print-air interface and the paper substrate and the lateral propagation of light within the paper bulk. However, the Clapper-Yule model assumes a planar interface and does not take into account the roughness of the print surface. In order to extend the ClapperYule model to rough printing supports (e.g., matte coated papers or calendered papers), we model the print surface as a set of randomly oriented microfacets. The influence of the shadowing effect is evaluated and incorporated into the model. By integrating over all incident angles and facet orientations, we are able to express the internal reflectance of the rough interface as a function of the rms facet slope. By considering also the rough interface transmittances both for the incident light and for the emerging light, we obtain a generalization of the Clapper-Yule model for rough interfaces. The comparison between the classical Clapper-Yule model and the model extended to rough surfaces shows that the influence of the surface roughness on the predicted reflectance factor is small. For high-quality papers such as coated and calendered papers, as well as for lowquality papers such as newsprint or copy papers, the influence of surface roughness is negligible, and the classical Clapper-Yule model can be used to predict the halftone-print reflectance factors. The influence of roughness becomes significant only for very rough and thick nondiffusing coatings. () 2005 Optical Society of America

OCIS codes: $100.2810,100.2810,240.5770,080.2710,100.2810,240.5770,160.0160,330.1710$.
\end{abstract}

\section{INTRODUCTION}

For more than 50 years, attempts have been made to build models predicting the color of printed halftone images. To offer accurate predictions, the models need to take into account the phenomena determining the interaction of light and halftone prints and of inks and paper.

Many different phenomena influence the reflection spectrum of a color halftone patch on a diffusely reflecting substrate (e.g., paper). These phenomena comprise the surface reflection (Fresnel reflectivity) at the interface between the air and the paper, light scattering and reflection within the substrate (i.e., paper bulk), and the internal reflections (average Fresnel reflectivity for a diffuse incident light) at the interface between the paper and the air. The lateral scattering of light within the paper substrate and the internal reflections at the interface between the paper and the air are responsible for what is generally called the optical dot gain (also known as the Yule-Nielsen effect).

Existing models for predicting the reflection spectra of color halftone patches rely on the Yule-Nielsen-modified spectral Neugebauer model, ${ }^{1-5}$ on the analysis of light propagation within the paper, ${ }^{6-9}$ or on the Clapper-Yule model. ${ }^{10}$

The Clapper-Yule model is the only classical model for halftone prints that takes explicitly into account both the multiple internal reflections between the paper substrate and the print-air interface and the lateral propagation of light within the paper bulk. Since the Clapper-Yule model assumes that lateral propagation of light within the printing support is significantly larger than the half- tone screen period, it is adapted to halftones printed only at high screen frequencies. However, extensions have been proposed for middle to low screen frequencies. ${ }^{11,12}$

The classical Clapper-Yule model assumes a perfectly smooth air-print interface. Only very glossy papers have a smooth interface. However, despite the fact that most prints have a rough surface yielding a matte appearance, the Clapper-Yule model yields excellent prediction results. ${ }^{13}$ We therefore establish a model extending the Clapper-Yule model to rough surfaces. Then we analyze the impact of the print surface roughness on the predicted spectral reflectance factor.

The reflectance of the halftone print characterizes the spectrum of the reflected light, according to a given measuring device geometry (illumination and detection orientations). A print is illuminated by an unpolarized collimated light beam oriented according to a fixed direction L. Two measuring geometries are thus considered: the L/diffuse geometry, where the spectral irradiance reflected by the print is collected by an integrating sphere, and the $\mathbf{L} / \mathbf{R}$ geometry, where a radiance detector captures the spectral radiance reflected by the print along a given direction R. Both geometries are supposed to discard the specular reflection at the surface of the print. We discard the specular reflection in the case of $\mathbf{L} / \mathbf{R}$ geometries by choosing asymmetric directions of $\mathbf{L}$ and $\mathbf{R}$ with respect to the print surface normal direction, e.g., an incident angle of $45^{\circ}$ and a viewing angle of $0^{\circ}\left(45^{\circ} / 0^{\circ}\right.$ geometry). Integrating spheres generally have a hole for discarding the specular reflection.

By dividing the reflection spectrum captured by a 
photospectrometer by the reflection spectrum of a reference white support captured under the same conditions, we obtain the spectral reflectance factor of the halftone print. We consider here two reference white supports: a perfectly white diffuse reflector, such as a barium sulfate white tile, and the unprinted printing support.

We can find in the literature several contributions about reflection or transmission of a light ray onto or through a rough interface between transparent media. ${ }^{14,15}$ These contributions may be useful for modeling the penetration of the incident light beam into the print. However, since they do not deal with the case of diffusely reflected light, they are neither suitable for calculating the interface's internal reflectance nor for characterizing the emergence of diffuse light from the print. Germer $^{16}$ proposes a model for incident light crossing a rough interface, being diffused by a substrate, undergoing multiple internal reflections, and crossing again the rough interface to the air. However, in contrast to our approach, he does not compute the internal reflectance of the rough interface but rather relies on a Monte Carlo simulation to average over all possible internal reflection directions.

We extend the Clapper-Yule model to rough air-print interfaces. This extension yields a closed-form expression allowing us to predict the reflection spectra of halftones printed on matte supports. We perform a detailed analysis of the impact of the interface roughness on the predicted spectral reflectance factors. This analysis confirms that, for high-quality matte papers, the classical ClapperYule model yields reflection spectra that are very close to the reflection spectra calculated thanks to the model extended to rough interfaces.

As an introduction to our model, we first recall the principles of the classical Clapper-Yule model for a planar air-print interface, by pursuing a strict radiometric approach (Section 2). In Section 3, we model the rough air-print interface, assuming that the rough interface average height and correlation length are larger than the wavelengths of the visible spectrum. This allows one to model the rough interface by a set of randomly oriented microfacets and to use the laws of geometrical optics. ${ }^{17}$ We show that the coating thickness has a direct impact on the light propagation between the paper bulk and the interface. The shadowing phenomenon, as well as multiple scattering between adjacent facets of the interface, is also considered. In Section 4, we deduce the internal reflectance coefficient as a function of the interface roughness and establish the closed-form expressions for the extended Clapper-Yule model. In Section 5, we evaluate the extended Clapper-Yule model as a function of the interface roughness for different combinations of measuring geometries and reference white supports. We draw the conclusions in Section 6 .

\section{CLAPPER-YULE MODEL FOR A PLANAR INTERFACE}

The halftone print is produced by depositing inked screen dots onto a printing support. The print's color is characterized by its wavelength-dependent reflectance factor, which can be predicted by the Clapper-Yule model if the printing support, the ink, and the periodic screen satisfy the following conditions.

The printing support is composed of a diffusely reflecting substrate having a flat interface with the air (e.g., glossy paper). The relative index of refraction $n_{\nu}$ of the air-print interface is the same in inked and in noninked regions, in both cases close to 1.5. The screen period is assumed to be small compared with the lateral propagation of light within the diffusing substrate. Therefore, the region (inked or noninked) from which the light penetrates the substrate is uncorrelated with the region (inked or noninked) toward which the light is reflected by the substrate.

The diffusing substrate is characterized by its spectral reflectance $\rho_{B}(\lambda)$, expressing the portion of irradiance that is reflected by the substrate. The ink layer is characterized by its spectral transmittance $t(\lambda)$, expressing the portion of irradiance crossing the ink layer without being absorbed.

Under these assumptions, the Clapper-Yule model describes the interaction of light with the halftone print (Subsection 2.A). It takes into account lateral propagation of light within the paper substrate and the multiple internal reflections that occur between the diffusing substrate and the print surface (print-air interface). In Subsection 2.B, we derive the expression of the halftone-print reflectance factor, depending on the measuring geometry and the selected reference white. We consider both the $\mathbf{L} /$ diffuse and the $\mathbf{L} / \mathbf{R}$ geometries. The incident light is a collimated beam arriving at an orientation $\mathbf{L}$. In the case of an $\mathbf{L} / \mathbf{R}$ geometry, the reflected light emerges at an orientation $\mathbf{R}$ and is captured by a radiance detector. We consider as reference white supports both a perfect white diffuse reflector and the unprinted paper support.

\section{A. Interaction of Light with a Planar Halftone Print}

The interaction of light with the halftone print can be separated into three components forming the ClapperYule model: the Fresnel transmittivity for the incident light crossing the air-print interface, the multiple reflections of the diffused light between the paper substrate and the print-air interface, and the Fresnel transmittivity for the emerging light crossing the print-air interface. All irradiances, radiances, and light fluxes are wavelength dependent (for the sake of simplicity, $\lambda$ is mentioned only on the first occurrence of a wavelengthdependent variable).

The print is illuminated by a collimated light of wavelength-dependent irradiance $V_{i}(\lambda)$. The air-print interface transmits an irradiance $W_{i}(\lambda)$ to the inked halftone layer and then to the paper bulk. An irradiance $W_{r}(\lambda)$, resulting from multiple reflections between the paper substrate and the print-air interface, is incident on the print-air interface. The print-air interface transmits an irradiance $V_{r}(\lambda)$ that can be captured by an integrating sphere or a radiance $L_{r}(\lambda)$ captured by a radiance detector (Fig. 1).

1. Transmission of the Incident Light through the Planar Air-Print Interface

The incident collimated beam of irradiance $V_{i}$, oriented according to vector $\mathbf{L}$, reaches the air-print interface of 


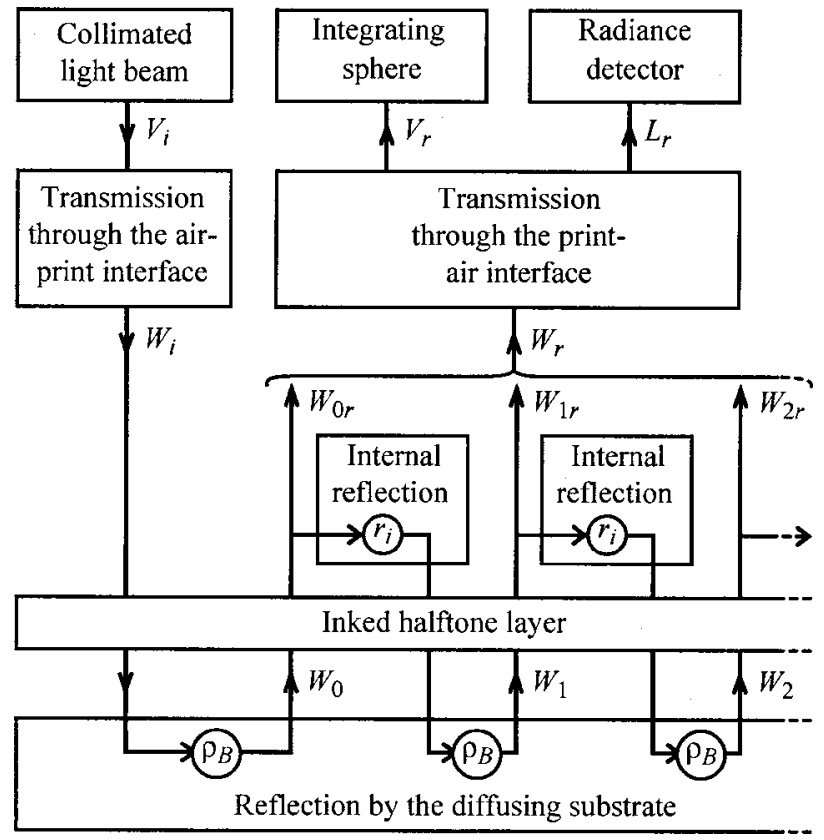

Fig. 1. Diagram of the interaction of light with the print according to the Clapper-Yule model.

normal vector $\mathbf{N}$. The ratio of light penetrating the print is given by the wavelength-independent Fresnel transmittivity $T_{n_{\nu}}(\mathbf{L}, \mathbf{N})$, where $n_{\nu}$ is the relative index of refraction of the air-print interface. ${ }^{18}$ The irradiance $W_{i}$ of the light transmitted through the interface is therefore

$$
W_{i}=T_{n_{\nu}}(\mathbf{L}, \mathbf{N}) V_{i}
$$

where $T_{n_{\nu}}(\mathbf{L}, \mathbf{N})$ is the transmittivity of the air-print interface.

\section{Multiple Internal Reflections between the Print-Air Interface and the Paper Substrate}

Having crossed once the air-print interface, the irradiance $W_{i}$ is subject to multiple internal reflections, during which light is alternately diffused and reflected by the paper substrate and by the print-air interface, until it is transmitted into the air across the print-air interface. The Clapper-Yule model assumes that lateral propagation of light within the paper bulk is large compared with the halftone screen period. Therefore, the probability of exiting from a given colorant is proportional to that colorant's surface coverage. The total irradiance $W_{r}$ that is incident onto the print side of the print-air interface is the sum of the elementary irradiances that reach the interface after each reflection by the paper bulk.

After having entered into the print, a part $a$ of irradiance $W_{i}$ traverses a colorant area of wavelengthdependent transmittance $t(\lambda)$, and a part $1-a$ traverses an uncolored area of transmittance 1, where $a$ is the fractional area covered by the colorant. The resulting irradiance is reflected back by the diffusing substrate of spectral reflectance $\rho_{B}(\lambda)$ toward the print-air interface:

$$
W_{0}=\rho_{B}(1-a+a t) W_{i} .
$$

This irradiance $W_{0}(\lambda)$ is decomposed into a first component $W_{0 r}(\lambda)$ that will be transmitted into the air through the print-air interface. This component $W_{0 r}$ reaches the print-air interface either in a colorant area (transmittance $t$, probability $a$ ) or in an uncolored area (transmittance 1 , probability $1-a$ ):

$$
W_{0 r}=(1-a+a t) W_{0} .
$$

A second component $W_{1}(\lambda)$ comprises the light that is internally reflected at the print-air interface (internal reflectance $r_{i}$ ), either in a colorant area (probability $a$, transmittance $t^{2}$ due to two passes through the ink layer) or in an uncolored area (probability $1-a$, transmittance 1 ), and then is reflected back by the substrate (reflectance $\left.\rho_{B}\right)$ :

$$
W_{1}=r_{i} \rho_{B}\left(1-a+a t^{2}\right) W_{0} .
$$

After $k$ internal reflections at the print-air interface, the irradiance $W_{k}(\lambda)$ is

$$
W_{k}=\left(r_{i} \rho_{B}\left(1-a+a t^{2}\right)\right)^{k} W_{0}, \quad k=1,2,3 \ldots .
$$

It is decomposed into an irradiance component $W_{k r}(\lambda)$ that will be transmitted into the air,

$$
W_{k r}=(1-a+a t) W_{k}, \quad k=1,2,3 \ldots,
$$

and a component $W_{k+1}(\lambda)$ that is internally reflected by the print-air interface and reflected back by the substrate. Finally, we obtain the total irradiance $W_{r}(\lambda)$ that will be transmitted through the print-air interface by summing all components $W_{k r}(\lambda)$. This yields the geometric series

$$
W_{r}=\sum_{k=0}^{\infty} W_{k r}=(1-a+a t)\left[\sum_{k=0}^{\infty}\left(r_{i} \rho_{B}\left(1-a+a t^{2}\right)\right)^{k}\right] W_{0},
$$

which, after Eq. (2) is inserted, converges toward

$$
W_{r}=\frac{\rho_{B}(1-a+a t)^{2}}{1-r_{i} \rho_{B}\left(1-a+a t^{2}\right)} W_{i} .
$$

In the same manner as Judd, ${ }^{19}$ let us compute the internal reflectance $r_{i}$ of the print-air interface. In Subsection 4.A we then generalize the internal reflectance to rough interfaces.

\section{Internal Diffuse Reflectance of a Planar Print-Air Interface}

The internal reflectance $r_{i}$ of an interface denotes the ratio of reflected to incident irradiances when a Lambertian diffuse light illuminates an element $d s$ of the interface. It corresponds to an average Fresnel reflectivity, taking into account all incidence directions of the rays that illuminate the interface. It is wavelength independent and depends only on the relative index of refraction $1 / n_{\nu}$ of the print-air interface.

An element $d s$ of the interface is illuminated by a Lambertian incident light of irradiance $E_{i}(\lambda)$. Therefore, it receives the radiance $E_{i} / \pi$ from each direction $\mathbf{V}$ of the hemisphere $\Omega_{\mathbf{N}}$, i.e., a flux element $d^{2} \Phi_{i}(\lambda)$ defined as 


$$
d^{2} \Phi_{i}=\frac{E_{i}}{\pi} d s\langle\mathbf{V} \cdot \mathbf{N}\rangle \mathrm{dV}_{\mathbf{N}}
$$

where the dot product $\langle\mathbf{V} \cdot \mathbf{N}\rangle$ designates the cosine of the angle between $\mathbf{V}$ and $\mathbf{N}$ and $d V_{\mathbf{N}}$ is the solid angle in which the flux is contained (see Appendix A). A part $F_{1 / n}(\mathbf{V}, \mathbf{N})$ of this incident flux element $d^{2} \Phi_{i}$ is reflected by the interface, yielding a reflected flux element $d^{2} \Phi_{r}(\lambda)$ :

$$
d^{2} \Phi_{r}=F_{1 / n_{\nu}}(\mathbf{V}, \mathbf{N}) \frac{E_{i}}{\pi} d s\langle\mathbf{V} \cdot \mathbf{N}\rangle \mathrm{dV}_{\mathbf{N}}
$$

By dividing both members of Eq. (7) by $d s$, we obtain the element of reflected irradiance $d^{2} \Phi_{r} / d s$, oriented according to direction $\mathbf{V}$ and contained within the solid angle $\mathrm{dV}_{\mathbf{N}}$. By integrating $d^{2} \Phi_{r} / d s$ according to $\mathrm{dV}_{\mathbf{N}}$ over the hemisphere $\Omega_{\mathrm{N}}$, we obtain the reflected irradiance $E_{r}(\lambda)$ :

$$
E_{r}=\frac{d \Phi_{r}}{d s}=\frac{E_{i}}{\pi} \int_{\Omega_{\mathbf{N}}} F_{1 / n_{\nu}}(\mathbf{V}, \mathbf{N})\langle\mathbf{V} \cdot \mathbf{N}\rangle \mathrm{dV}_{\mathbf{N}} .
$$

The ratio of the reflected irradiance $E_{r}$ to the incident irradiance $E_{i}$ yields the internal reflectance $r_{i}$ of the interface, which is wavelength independent:

$$
r_{i}=\frac{1}{\pi} \int_{\Omega_{\mathbf{N}}} F_{1 / n_{\nu}}(\mathbf{V}, \mathbf{N})\langle\mathbf{V} \cdot \mathbf{N}\rangle \mathrm{dV}_{\mathbf{N}} .
$$

Internal reflectance coefficient $r_{i}$ can be expressed by an equivalent angular formulation. If $(\theta, \phi)$ are the polar and azimuthal coordinates of vector $\mathbf{V}$ defined with respect to $\mathbf{N}$, we can replace $\langle\mathbf{V} \cdot \mathbf{N}\rangle$ with $\cos \theta$ and $\mathrm{dV}_{\mathbf{N}}$ with $\sin \theta \mathrm{d} \theta \mathrm{d} \phi$. We have

$$
r_{i}=\frac{1}{\pi} \int_{\phi=0}^{2 \pi} \int_{\theta=0}^{\pi / 2} F_{1 / n_{\nu}}(\theta) \cos \theta \sin \theta \mathrm{d} \theta \mathrm{d} \phi .
$$

Since the Fresnel factor does not depend on the azimuth angle $\phi$, the integration according to $\phi$ yields a factor of $2 \pi$. Equation (9) becomes

$$
r_{i}=\int_{\theta=0}^{\pi / 2} F_{1 / n_{\nu}}(\theta) \sin 2 \theta \mathrm{d} \theta .
$$

According to the principle of conservation of energy, the transmittance coefficient of the print-air interface is

$$
t_{i}=1-r_{i}
$$

\section{Transmission of the Emerging Irradiance through the Planar Print-Air Interface}

The irradiance emerging from the print is given by the product of the interface's transmittance [Eq. (11)] and the irradiance $W_{r}$ incident at the print side of the interface [Eq. (5)]:

$$
V_{r}=\left(1-r_{i}\right) W_{r} .
$$

The irradiance $V_{r}$ emerges from the print over the whole upper hemisphere and may, for example, be captured by an integrating sphere. By inserting Eqs. (1) and (5) into Eq. (12), we find that the expression of the emerg- ing irradiance $V_{r}$ corresponds to the product of the three components of the Clapper-Yule model with the incident irradiance $V_{i}$ :

$$
V_{r}=T_{n_{\nu}}(\mathbf{L}, \mathbf{N}) \cdot \frac{\rho_{B}(1-a+a t)^{2}}{1-r_{i} \rho_{B}\left(1-a+a t^{2}\right)} \cdot\left(1-r_{i}\right) V_{i} .
$$

In expression (13), component $T_{n_{\nu}}(\mathbf{L}, \mathbf{N})$ expresses the Fresnel transmittivity of the air-print interface for the incident light, the central fraction expresses the attenuation due to the multiple internal reflections, and the component $\left(1-r_{i}\right)$ expresses the average Fresnel transmittivity of the print-air interface for the diffuse emerging light.

\section{Transmission of the Radiance Emerging in the} Direction of a Radiance Detector

Because of the angular dependence of the Fresnel transmittivity, the irradiance $V_{r}$ emerging from the print is not Lambertian, in contrast to the incident irradiance $W_{r}$. For that reason, the radiance $L_{r}$ measured by a radiance detector cannot be derived directly from $V_{r}$. It is calculated thanks to the Fresnel transmittivity by considering explicitly the measuring direction $\mathbf{R}$.

Radiance $L_{r}(\lambda)$ is the flux element $d^{2} \Phi(\lambda)$ that the detector receives along the normal of its surface $d s_{d}$ within its solid angle $d_{R_{\mathbf{R}^{-}}^{-}}$(Fig. 2). According to the radiance invariance principle,${ }^{20} L_{r}$ is also the flux element $d \Phi$ transmitted by an element $d s$ of the air-print interface and contained within a solid angle $\mathrm{dR}_{\mathrm{N}}=\sin \theta_{r} \mathrm{~d} \theta_{r} \mathrm{~d} \phi_{r}$ oriented according to the direction $\mathbf{R}$ of the radiance detector:

$$
L_{r}=\frac{d^{2} \Phi}{d s_{d} \mathrm{dR}_{\mathbf{R}^{-}}^{-}}=\frac{d^{2} \Phi}{d s\langle\mathbf{R} \cdot \mathbf{N}\rangle \mathrm{dR}_{\mathbf{N}}},
$$

where $d s\langle\mathbf{R} \cdot \mathbf{N}\rangle$ is the projected area of the interface element $d s$ along direction $\mathbf{R}$. The emerging flux element

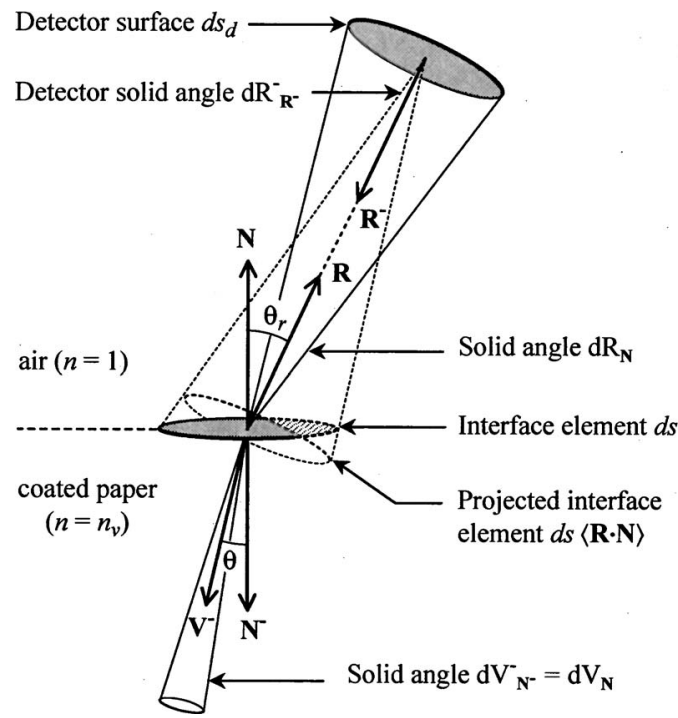

Fig. 2. Element $d s$ of a planar interface, of normal vector $\mathbf{N}$, receives a radiance from direction $\mathbf{V}^{-}$, within a solid angle $\mathrm{dV}_{\mathbf{N}^{-}}^{-}$ $=\mathrm{dV}_{\mathbf{N}}=\sin \theta \mathrm{d} \theta \mathrm{d} \phi$. The radiance transmitted into the air, in direction $\mathbf{R}$ within a solid angle $\mathrm{dR}_{\mathbf{N}}=\sin \theta_{r} \mathrm{~d} \theta_{r} \mathrm{~d} \phi_{r}$, is equal to the radiance received by the surface $d s_{d}$ of the detector within its solid angle $\mathrm{dR}_{\mathbf{R}^{-}}^{-}$. Vectors $\mathbf{V}, \mathbf{N}$, and $\mathbf{R}$ are related according to Snell's refraction law. 
$d^{2} \Phi$ is the transmitted part $T_{n_{\nu}}(\mathbf{R}, \mathbf{N})$ of the flux element $d^{2} \Phi_{i}(\lambda)$ that is incident onto the print side of the interface:

$$
d^{2} \Phi=T_{n_{\nu}}(\mathbf{R}, \mathbf{N}) d^{2} \Phi_{i} .
$$

The direction $\mathbf{R}\left(\theta_{r}, \phi_{r}\right)$ of the transmitted flux element $d^{2} \Phi$ and the direction $\mathbf{V}(\theta, \phi)$ of the incident flux element $d^{2} \Phi_{i}$ are related by Snell's refraction laws:

$$
\begin{gathered}
\phi_{r}=\phi, \\
\sin \theta_{r}=n_{\nu} \sin \theta .
\end{gathered}
$$

The flux element $d^{2} \Phi_{i}$ incident onto $d s$ from direction $\mathbf{V}$ through the solid angle $\mathrm{dV}_{\mathbf{N}}=\sin \theta \mathrm{d} \theta \mathrm{d} \phi$ yields a radiance equal to $W_{r} / \pi$, since the irradiance $W_{r}$ is Lambertian:

$$
\frac{W_{r}}{\pi}=\frac{d^{2} \Phi_{i}}{d s\langle\mathbf{V} \cdot \mathbf{N}\rangle \mathrm{dV}_{\mathbf{N}}}
$$

The relation between the term $\langle\mathbf{R} \cdot \mathbf{N}\rangle \mathrm{dR}_{\mathbf{N}}$ appearing in Eq. (14) and the term $\langle\mathbf{V} \cdot \mathbf{N}\rangle \mathrm{dV}_{\mathbf{N}}$ appearing in Eq. (17) can be calculated by considering the differential of expressions (16):

$$
\begin{gathered}
\mathrm{d} \phi_{r}=\mathrm{d} \phi, \\
\cos \theta_{r} \mathrm{~d} \theta_{r}=n_{\nu} \cos \theta \mathrm{d} \theta .
\end{gathered}
$$

Then, according to Fig. 2 and by considering Eqs. (16) and (18), we have

$$
\frac{\langle\mathbf{V} \cdot \mathbf{N}\rangle \mathrm{dV}_{\mathbf{N}}}{\langle\mathbf{R} \cdot \mathbf{N}\rangle \mathrm{dR}_{\mathbf{N}}}=\frac{\cos \theta \sin \theta \mathrm{d} \theta \mathrm{d} \phi}{\cos \theta_{r} \sin \theta_{r} \mathrm{~d} \theta_{\mathrm{r}} \mathrm{d} \phi_{r}}=\frac{1}{n_{\nu}^{2}} .
$$

By inserting Eqs. (15), (17), and (19) into the expression of $L_{r}$ in Eq. (14), we obtain

$$
L_{r}=T_{n_{\nu}}(\mathbf{R}, \mathbf{N}) \frac{1}{n_{\nu}^{2}} \frac{W_{r}}{\pi}
$$

where the expression of $W_{r}$ is given by combining Eqs. (1) and (5). Finally, the radiance $L_{r}$ emerging from the print in the direction $\mathbf{R}$ of the radiance detector is

$$
L_{r}=T_{n_{\nu}}(\mathbf{L}, \mathbf{N}) \cdot \frac{\rho_{B}(1-a+a t)^{2}}{1-r_{i} \rho_{B}\left(1-a+a t^{2}\right)} \cdot \frac{T_{n_{\nu}}(\mathbf{R}, \mathbf{N})}{n_{\nu}^{2}} \frac{V_{i}}{\pi} .
$$

\section{B. Expressing the Clapper-Yule Reflectance Factor for a Planar Interface}

The expressions for the reflectance factor predicted with the Clapper-Yule model depend on how the emerging light is captured, i.e., either by an integrating sphere (L/diffuse geometry) or by a radiance detector $(\mathbf{L} / \mathbf{R}$ geometry). They also depend on the chosen reference white (perfectly white diffuse reflector or unprinted printing support).

\section{L/Diffuse Geometry}

The reflectance of the halftone print is the ratio of the irradiance $V_{r}$ captured by the integrating sphere to the irradiance $V_{i}$ of the incident light beam. It is derived directly from Eq. (13).

The reflectance factor $R_{\Omega w}(\lambda)$ defined in reference to a perfectly white diffuse reflector is obtained by dividing the print's reflectance $V_{r} / V_{i}$ by the reflectance of the perfectly white diffuse reflector, which is 1 at all wavelengths of the visible range:

$$
R_{\Omega w}=T_{n_{\nu}}(\mathbf{L}, \mathbf{N})\left(1-r_{i}\right) \frac{\rho_{B}\left(1-a+a t_{\lambda}\right)^{2}}{1-r_{i} \rho_{B}\left(1-a+a t_{\lambda}^{2}\right)} .
$$

The reflectance factor $R_{\Omega p}(\lambda)$ defined in reference to the unprinted printing support is obtained by dividing the print's reflectance $V_{r} / V_{i}$ by the reflectance of the unprinted paper support. Under the condition that the integrating sphere discards the light specularly reflected at the air-print interface, the terms expressing the Fresnel transmittivity for the penetrating and emerging light are canceled out:

$$
R_{\Omega p}=\frac{V_{r} / V_{i}}{V_{r}(a=0) / V_{i}}=\frac{\left(1-r_{i} \rho_{B}\right)(1-a+a t)^{2}}{1-r_{i} \rho_{B}\left(1-a+a t^{2}\right)} .
$$

Expressions (22) and (23) are given in Clapper and Yule's original paper. ${ }^{10}$

\section{2. $\boldsymbol{L} / \boldsymbol{R}$ geometry}

The radiance detector captures the radiance $L_{\nu}$ given by Eq. (21). A reflectance factor is defined as a ratio of reflectances (print's reflectance to reference reflectance). It can also be defined as a ratio of radiances if the illuminating and measuring conditions are the same for the halftone print and for the reference support. Therefore, the reflectance factor $R_{\delta w}(\lambda)$ defined in reference to a perfectly white diffuse reflector is obtained by dividing $L_{r}$ by the radiance $L_{\text {ref }}(\lambda)=V_{i} / \pi$ measured by the radiance detector from a perfectly white diffuse reflector:

$$
R_{\delta w}=T_{n_{\nu}}(\mathbf{L}, \mathbf{N}) \frac{T_{n_{\nu}}(\mathbf{R}, \mathbf{N})}{n_{\nu}^{2}} \frac{\rho_{B}(1-a+a t)^{2}}{1-r_{i} \rho_{B}\left(1-a+a t^{2}\right)} .
$$

If we choose as the reference the unprinted printing support, we divide the radiance $L_{r}$ by the radiance that the detector captures from the unprinted printing support, which is derived from Eq. (21), with $a=0$. The corresponding reflectance factor $R_{\delta p}(\lambda)$ is

$$
R_{\delta p}=\frac{L_{r}}{L_{r}(a=0)}=\frac{\left(1-r_{i} \rho_{B}\right)(1-a+a t)^{2}}{1-r_{i} \rho_{B}\left(1-a+a t^{2}\right)} .
$$

Since $R_{\Omega p}$ and $R_{\delta p}$ have the same expression, the reflectance factors defined in reference to the unprinted printing support are therefore identical for both the L/diffuse and the $\mathbf{L} / \mathbf{R}$ geometries.

The classical Clapper-Yule model assumes that the air-print interface is perfectly smooth. It is therefore well adapted for modeling the interaction of light with halftones printed on glossy printing supports, such as glossy coated papers. In practice, however, the Clapper-Yule model is often applied to matte printing supports having 
a rough surface and still seems to provide excellent predictions. We propose therefore an extension of the Clapper-Yule model in order to predict the spectral reflectance factor of halftones printed on matte printing supports. This extended model allows us to precisely quantify to what extent the spectral reflectance factors depend on the roughness of the print-air interface.

\section{MODELING THE ROUGH AIR-PRINT INTERFACE}

The rough air-print interface is statistically described by a set of randomly inclined microfacets, whose inclination is parameterized by the standard deviation $m$ of the interface facet slopes (Subsection 3.A). In Subsection 3.B we show that the illumination range of the rough interface by the diffusing substrate depends on the substrate-tointerface distance. In Subsection 3.C we introduce Smith's shadowing model, which expresses the illumination attenuation due to mutual shadowing at nearby interface facets.

\section{A. Microfacet Model of the Rough Air-Print Interface} The rough interface is modeled by a set of randomly inclined facets, in a manner similar to the TorranceSparrow microfacet model. ${ }^{21} \mathrm{~A}$ facet inclination may be described by its slope $s$ as well as by its normal vector $\mathbf{H}$. The facet slope is statistically specified by a probability distribution function. In order to apply the laws of geometrical optics (Snell's laws, Fresnel formulas), we are interested in describing the inclination of facets according to their normal vector. By determining the relationship between the facet slope $s$ and the facet normal vector $\mathbf{H}$, we can convert the slope distribution function into an equivalent distribution function of the polar and azimuthal angles characterizing the facet normal vector $\mathbf{H}$.

Let us consider a facet normal vector $\mathbf{H}$, specified by its spherical angles $\left(\theta_{h}, \phi_{h}\right)$ and by its corresponding Cartesian coordinates $\left(\sin \theta_{h} \cos \phi_{h}, \sin \theta_{h} \sin \phi_{h}, \cos \theta_{h}\right)$. A facet of normal $\mathbf{H}$ can be assimilated to an inclined plane in the $(x, y, z)$ Cartesian space, of the equation

$$
\left(\sin \theta_{h} \cos \phi_{h}\right) x+\left(\sin \theta_{h} \sin \phi_{h}\right) y+\left(\cos \theta_{h}\right) z=0 .
$$

Let us express $z$ as a function of $x$ and $y$ :

$$
z=-\left(\tan \theta_{h} \cos \phi_{h}\right) x-\left(\tan \theta_{h} \sin \phi_{h}\right) y .
$$

The derivative of $z$ along $x$ yields the slope component $s_{x}$ of the plane along the $x$ axis; similarly, the derivative of $z$ along $y$ yields the slope component $s_{y}$ of the plane along the $y$ axis:

$$
\begin{aligned}
& s_{x}=\partial z / \partial x=-\tan \theta_{h} \cos \phi_{h}, \\
& s_{y}=\partial z / \partial y=-\tan \theta_{h} \sin \phi_{h} .
\end{aligned}
$$

The maximum slope of the plane (modulus of the gradient vector) is

$$
s=\sqrt{s_{x}^{2}+s_{y}^{2}}=\tan \theta_{h} .
$$

These slope components $s_{x}$ and $s_{y}$ are typically assumed to be independent random variables following a Gaussian distribution $f$ parameterized by the standard deviation $m$ of the slope of the rough interface (also called rms slope):

$$
f\left(s_{x}\right)=\frac{\exp \left(-s_{x}^{2} / 2 m^{2}\right)}{\sqrt{2 \pi} m}, \quad f\left(s_{y}\right)=\frac{\exp \left(-s_{y}^{2} / 2 m^{2}\right)}{\sqrt{2 \pi} m} .
$$

A given facet has a probability $P\left(s_{x}\right)=f\left(s_{x}\right) \mathrm{d} s_{x}$ to have a slope component in direction $x$ between $s_{x}$ and $s_{x}+\mathrm{d} s_{x}$ and a probability $P\left(s_{y}\right)=f\left(s_{y}\right) \mathrm{d} s_{y}$ to have a slope component in direction $y$ between $s_{y}$ and $s_{y}+\mathrm{d} s_{y}$. The joint probability for this facet, per unit differential slope $\mathrm{d} s_{x} \mathrm{~d} s_{y}$, to have an effective slope $s=\sqrt{s_{x}^{2}+s_{y}^{2}}$ is therefore

$$
P(s)=P\left(s_{x}\right) P\left(s_{y}\right)=\frac{\exp \left(-s^{2} / 2 m^{2}\right)}{2 \pi m^{2}} \mathrm{~d} s_{x} \mathrm{~d} s_{y} .
$$

In order to express this probability as a function of the polar and azimuthal angles $\left(\theta_{h}, \phi_{h}\right)$ of the facet's normal vector $\mathbf{H}$, per unit solid angle $\mathrm{dH}_{\mathbf{N}}=\sin \theta_{h} \mathrm{~d} \theta_{h} \mathrm{~d} \phi_{h}$, we carry out a change of variables. With Eqs. (28), the Jacobian of this change of variables is

$\frac{\mathrm{d} s_{x} \mathrm{~d} s_{y}}{\mathrm{dH}_{\mathbf{N}}}=\frac{\mathrm{d} s_{x} \mathrm{~d} s_{y}}{\sin \theta_{h} \mathrm{~d} \theta_{h} \mathrm{~d} \phi_{h}}=\frac{1}{\sin \theta_{h}}\left|\begin{array}{ll}\partial s_{x} / \partial \theta_{h} & \partial s_{x} / \partial \phi_{h} \\ \partial s_{y} / \partial \theta_{h} & \partial s_{y} / \partial \phi_{h}\end{array}\right|=\frac{1}{\cos ^{3} \theta_{h}}$.

By replacing, in Eq. (31), $s$ with $\tan \theta_{h}$ and $\mathrm{d} s_{x} \mathrm{~d} s_{y}$ with $\mathrm{dH}_{\mathbf{N}} / \cos ^{3} \theta_{h}$ (Eq. (32)), we can express the probability $P(\mathbf{H})$ that the normal $\mathbf{H}$ of a facet belongs to the solid angle $\mathrm{dH}_{\mathbf{N}}$ :

$$
P(\mathbf{H})=D(\mathbf{H}) \mathrm{dH}_{\mathbf{N}},
$$

where $D(\mathbf{H})$ is the probability distribution function of the facet's normal vector $\mathbf{H}$ :

$$
D(\mathbf{H})=\frac{\exp \left(-\tan ^{2} \theta_{h} / 2 m^{2}\right)}{2 \pi m^{2} \cos ^{3} \theta_{h}} .
$$

The expression of $D(\mathbf{H})$ depends only on the polar angle $\theta_{h}$ of $\mathbf{H}$. This is characteristic of the roughness azimuthal isotropy that was implied by considering the same rms slope $m$ in the $x$ and $y$ directions. One may verify that the integration of $D(\mathbf{H})$ over the hemisphere $\Omega_{\mathrm{N}}$ yields

$$
\int_{\Omega_{\mathbf{N}}} D(\mathbf{H}) \mathrm{dH}_{\mathbf{N}}=1 .
$$

Note that the planar interface is the limit case of a rough interface with a $\mathrm{rms}$ slope $m=0$. The distribution function $D(\mathbf{H})$ of a planar interface becomes the Dirac delta function $\delta(\mathbf{N})$.

\section{B. Illumination by the Diffusing Substrate According to the Substrate-Interface Distance}

The diffusing substrate, which reflects light toward the interface, can be considered a diffuse light source illuminating the facets composing the rough interface. Light rays emitted by this source are reflected and refracted by the rough interface according to their propagation direction. In contrast to planar interfaces whose illumination range is necessarily the upper hemisphere $\Omega_{N}$, rough in- 


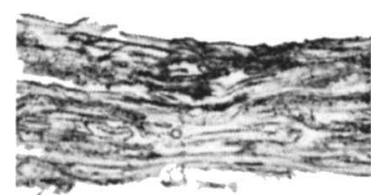

(a)

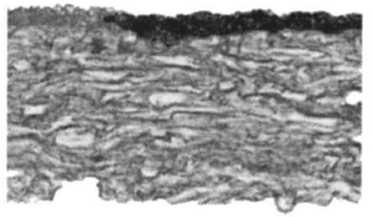

(b)

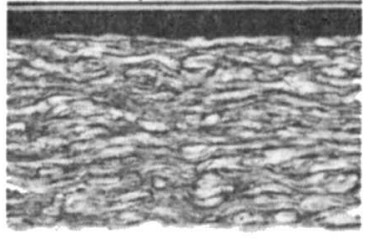

(c)

Fig. 3. (a) Cross section of an ink-jet print on uncoated paper (paper thickness of $106 \mu \mathrm{m}$ ), (b) cross section of an ink-jet print on coated paper (paper thickness of $118 \mu \mathrm{m}$ ), (c) cross section of an ink-jet print on resin-coated paper (paper thickness of $157 \mu \mathrm{m}$ ); courtesy of Becker and Kasper. ${ }^{22}$

terfaces may have a larger illumination range, depending on the distance between the diffusing substrate and the print-air interface.

The substrate-interface distance is considered to be large when the minimal elevation of the rough interface (i.e., the grooves' bottom) is higher than the maximal elevation of the diffusing substrate. In such a case, a substrate-to-interface straight path is possible only along a direction included within the upper hemisphere. The illumination range of a rough interface located at a large distance from the substrate is therefore $\Omega_{\mathbf{N}}$.

In the opposite case, the substrate is in contact with the interface. The facets are therefore completely illuminated, over their full hemisphere. Since facet orientations can be up to $90^{\circ}$ (vertical facets), substrate-to-interface straight paths are possible along the $-\mathbf{N}$ direction (incidence angle of $180^{\circ}$ ). The illumination range of a rough hemisphere at a short distance from the substrate is therefore the full sphere $\Sigma_{\mathbf{N}}$. Intermediate substrateinterface distances obviously yield intermediate illumination ranges.

Examples of short and long substrate-interface distances are shown in Fig. 3. Figure 3(a) shows an uncoated paper, whose diffusing substrate is in contact with the rough print-air interface. Figure $3(\mathrm{~b})$ shows a coated paper, where the thick coating layer is responsible for the large substrate-to-interface distance. Figure 3(c) shows a resin-coated paper that has been laminated after being printed, yielding a smooth print-air interface. Without the laminating process, the air-print interface would have been slightly rough as on the back side of the paper. Here also, the diffusing substrate is at a large distance from the print-air interface.

\section{Shadowing}

In contrast to a planar interface, which is equally illuminated at each point whatever the illumination condition, a rough interface may comprise shadow areas that increase when one increases the interface roughness and the illumination incidence angle. Interface elements belonging to shadow areas do not contribute to the reflected and transmitted irradiances.

Smith's shadowing model ${ }^{23}$ calculates the probability $G_{m}(\mathbf{V}, \mathbf{H})$ that a given interface element of normal vector $\mathbf{H}$ is illuminated, given the illumination direction $\mathbf{V}$ and the rms slope $m$ of the surrounding interface elements. When $\mathbf{V}$ does not belong to the hemisphere $\Omega_{\mathbf{H}}$ oriented according to vector $\mathbf{H}$ (i.e., the angle between $\mathbf{V}$ and $\mathbf{H}$ exceeds $\pi / 2$ and $\langle\mathbf{V} \cdot \mathbf{H}\rangle$ is negative), the facets of normal

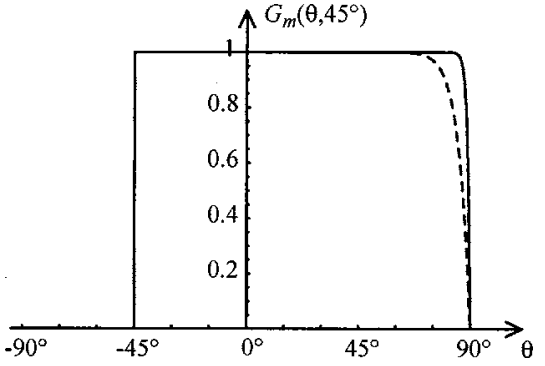

Fig. 4. Smith's illumination probability function for interface's rms slopes of $m=0.05$ (solid curve) and $m=0.2$ (dashed curve). The illumination probability of facets inclined with an angle of $45^{\circ}$ is plotted as a function of the light incidence angle $\theta$. The illumination probability is zero for incident angles $\theta<-45^{\circ}$.

vector $\mathbf{H}$ cannot be illuminated, and $G_{m}(\mathbf{V}, \mathbf{H})$ is equal to zero. Otherwise, $G_{m}(\mathbf{V}, \mathbf{H})$ is between 0 (facets completely shadowed) and 1 (facets completely illuminated):

$$
G_{m}(\mathbf{V}, \mathbf{H})=\frac{h(\langle\mathbf{V} \cdot \mathbf{H}\rangle)}{\Lambda_{m}(\theta)+1}=\left\{\begin{array}{ll}
\frac{1}{\Lambda_{m}(\theta)+1} & \left(\text { if } \mathbf{V} \in \Omega_{\mathbf{H}}\right) \\
0 & \left(\text { if } \mathbf{V} \notin \Omega_{\mathbf{H}}\right)
\end{array},\right.
$$

where $\theta$ is the light's incidence angle (angle between $\mathbf{V}$ and $\mathbf{N}$ ), $h(x)$ is the unit step function ( 0 for $x$ negative and 1 for $x$ positive), and

$$
\Lambda_{m}(\theta)=\frac{1}{2}\left[\frac{1}{\sqrt{\pi}} \frac{\sqrt{2} m}{\cot \theta} \exp \left(-\frac{\cot ^{2} \theta}{2 m^{2}}\right)-\operatorname{erfc}\left(\frac{\cot \theta}{\sqrt{2} m}\right)\right] .
$$

When the interface roughness is increased, facets are shadowed by higher-slope neighboring interface elements, and therefore at high incidence angles the illumination probability $G_{m}(\mathbf{V}, \mathbf{H})$ decreases. In the example of Fig. 4, the illumination probability $G_{m}\left(\theta, 45^{\circ}\right)$ of facets inclined at $45^{\circ}$ [i.e., angle $(\mathbf{H}, \mathbf{N})=45^{\circ}$ ] is shown. At high incidence angles, for a rough interface ( $\mathrm{rms}$ slope $m=0.2$ ), the illumination probability decreases more strongly than for a smooth interface $(m=0.05)$.

At small and medium incidence angles, the illumination probability function is close to 1 . The shadowing effect is thus small enough to be neglected. However, if we ignore the shadowing at high incidence angles, we may overestimate the reflected and transmitted irradiances and therefore violate the principle of conservation of en- 
ergy. Therefore, shadowing needs to be taken into account, according to Bruce ${ }^{24}$ and Caron et al. ${ }^{15}$ when the incidence angle is higher than a limit angle $\theta_{\text {shad }}$ depending on the rms slope $m$ of the rough interface:

$$
\theta_{\text {shad }}=\frac{\pi}{2}-\arctan (\sqrt{2} m)
$$

The shadowing effect also occurs when the rough interface is viewed under a given angle by a radiance detector. For viewing angles higher than the limit angle $\theta_{\text {shad }}$, the illumination probability function $G_{m}$ should be used, with vector $\mathbf{V}$ denoting the direction of observation instead of the direction of illumination.

As in other contributions on light scattering of rough interfaces, ${ }^{14,15}$ we ignore cases where a light ray undergoes several successive reflections or refractions at different interface elements, since the product of the corresponding Fresnel reflectivities or transmittivities or both is close to zero.

With the criterion of Eq. (37), let us describe when the shadowing effect is to be included in our extended Clapper-Yule model. Hansson ${ }^{25}$ has shown, thanks to profilometric measurements performed on several representative paper types, that the rms slope of most paper surfaces does not exceed 0.2. Such a maximal rms slope yields a worse-case limit angle $\theta_{\text {shad }}$ of $74^{\circ}$.

In order to avoid the shadowing effect at the air side of the interface, we assume that the direction $\mathbf{L}$ of the incident light forms an angle $\theta_{\mathbf{L}}<74^{\circ}$ (for example, $45^{\circ}$ as in the $45^{\circ} / 0^{\circ}$ geometry). We can neglect the shadowing effect on the coating side of the interface, since the rough interface scatters the incident light into a range of angles centered around the refraction angle $\left(27.5^{\circ}\right.$ in the example of $\theta_{\mathbf{L}}=45^{\circ}$ ), which is a fortiori lower than $74^{\circ}$, since $\arcsin \left(\sin \theta_{\mathbf{L}} / n_{\nu}\right) \leqslant \theta_{\mathbf{L}}<\theta_{\text {shad }}$.

The diffuse light reflected by the diffusing substrate is either internally reflected at the rough interface or transmitted and captured by the integrating sphere. In the case where the diffusing substrate is close to the rough interface, since the facets are completely illuminated (see Subsection 3.B), the shadowing effect does not exist on the coating side of the interface. In the case of a large substrate-interface distance, since the incident diffuse light comprises propagation directions exceeding $\theta_{\text {shad }}$, Smith's illumination probability function $G_{m}$ [Eq. (36)] needs to be included for calculating the rough interface's internal reflectance.

With respect to the diffuse emerging light captured by an integrating sphere, shadowing occurs at the air side of the rough interface for high exit angles, at which the corresponding Fresnel transmittivity is close to zero, or for high-slope facets, which have a very low occurrence probability. We therefore ignore shadowing at the air side of the rough interface for diffuse emerging light, in the cases of both small and large substrate-to-interface distances.

With respect to the emerging light captured by a radiance detector, the same considerations as for the incident light apply. We therefore avoid the shadowing effect by selecting for the radiance detector a viewing angle $\theta_{\mathbf{R}}$ inferior to $74^{\circ}$.

\section{EXTENDED CLAPPER-YULE MODEL FOR A ROUGH INTERFACE}

We extend the Clapper-Yule model presented in Section 2 to rough air-print interfaces, whose facet inclinations follows a Gaussian distribution (Section 3). We express for a rough print interface the irradiance $V_{r}^{\prime}(\lambda)$ captured by an integrating sphere and the radiance $L_{r}^{\prime}(\lambda)$ captured by a radiance detector and then derive the reflectance factors related to each measuring geometry.

The irradiance of the incident light beam $V_{i}^{\prime}(\lambda)$, the emerging irradiance $V_{r}^{\prime}(\lambda)$, and the emerging radiance $L_{r}^{\prime}(\lambda)$ as well as the intermediate irradiances $W_{i}^{\prime}(\lambda)$ and $W_{r}^{\prime}(\lambda)$ for a rough interface are defined in the same way as the corresponding variables $V_{i}(\lambda), V_{r}(\lambda), L_{r}(\lambda), W_{i}(\lambda)$, and $W_{r}(\lambda)$ of the planar interface (Fig. 1).

\section{A. Interaction of Light with a Rough Halftone Print} The random slope of the facets composing the rough print-air interface follows a Gaussian distribution of standard deviation $m$. The surface topology, i.e., the facet slope distribution, is assumed to be the same in inked and noninked areas.

\section{Transmission of the Incident Light through the Rough Air-Print Interface}

The incident light beam illuminates the facets composing the rough air-print interface with an irradiance $V_{i}^{\prime}$. The incidence direction $\mathbf{L}$ is chosen so as to ensure that the incidence angle between $\mathbf{L}$ and $\mathbf{N}$ is lower than the limit angle $\theta_{\text {shad }}$ [Eq. (37)]. We can therefore ignore the shadowing effect and assume that all facets are completely illuminated.

The facets contribute to the transmitted irradiance $W_{i}^{\prime}$ in different proportions according to their orientation. Given an orientation $\mathbf{H}$, the rough interface comprises a fraction $P(\mathbf{H})$ of facets with normal vector $\mathbf{H}$, which receive an irradiance $V_{i}^{\prime} P(\mathbf{H})$. Since this fraction of facets has a Fresnel transmittivity $T_{n_{\nu}}(\mathbf{L}, \mathbf{H})$, it transmits an irradiance $V_{i}^{\prime} P(\mathbf{H}) T_{n_{\nu}}(\mathbf{L}, \mathbf{H})$. By considering all normal vectors $\mathbf{H}$ within the upper hemisphere, we sum their contributions $V_{i}^{\prime} P(\mathbf{H}) \quad T_{n_{\nu}}(\mathbf{L}, \mathbf{H})$ and obtain the total transmitted irradiance $W_{i}^{\prime}$. After replacing $P(\mathbf{H})$ with $D(\mathbf{H}) \mathrm{dH}_{\mathbf{N}}$ according to Eq. (33), we obtain

$$
W_{i}^{\prime}=V_{i}^{\prime} \int_{\Omega_{\mathbf{N}}} T_{n_{\nu}}(\mathbf{L}, \mathbf{H}) D(\mathbf{H}) \mathrm{dH}_{\mathbf{N}} .
$$

Equation (38) is the generalization of Eq. (1). Let us call $\tau_{n_{\nu}}(\mathbf{L})$ the transmittance of the rough interface given by the ratio $W_{i}^{\prime} / V_{i}^{\prime}$ :

$$
\tau_{n_{\nu}}(\mathbf{L})=\int_{\Omega_{\mathbf{N}}} T_{n_{\nu}}(\mathbf{L}, \mathbf{H}) D(\mathbf{H}) \mathrm{dH}_{\mathbf{N}} .
$$

The transmittance of the rough interface $\tau_{n_{y}}(\mathbf{L})$ depends only on the light incidence direction $\mathbf{L}$ and on the relative index of refraction $n_{\nu}$ of the interface. It is therefore wavelength independent. It represents the average Fresnel transmittivity of the rough interface, where the contribution of each facet orientation is weighted by the probability of its orientation. 


\section{Multiple Internal Reflections}

As in the case of a planar interface, the irradiance $W_{i}^{\prime}$ is subject to multiple internal reflections, during which light rays are alternately reflected by the diffusing substrate and by the rough print-air interface. The roughness of the interface has no influence on the substrate reflectance $\rho_{B}$, the ink coverage $a$, and the ink transmittance $t$. It influences only the internal reflectance $\bar{r}_{i}$ of the print-air interface. Therefore, the irradiance $W_{r}^{\prime}$ that reaches the interface after multiple reflections takes the same expression as in Eq. (5), where the internal reflectance of the planar interface $r_{i}$ is replaced by the internal reflectance of the rough interface $\bar{r}_{i}$ :

$$
W_{r}^{\prime}=\frac{\rho_{B}(1-a+a t)^{2}}{1-\bar{r}_{i} \rho_{B}\left(1-a+a t^{2}\right)} W_{i}^{\prime} .
$$

The rough interface internal reflectance $\bar{r}_{i}$, presented in detail in Subsection 4.A.3, depends on the interface relative index of refraction (as for a flat interface), on the interface roughness, and on the distance between the interface and the diffusing substrate.

\section{Internal Reflectance of the Rough Interface}

The internal reflectance $\bar{r}_{i}$ is the ratio of a diffuse incident irradiance that is reflected at the print side of the rough air-print interface. It represents an average Fresnel reflectivity, taking into account the propagation directions of the incident diffuse light (illumination range) and the inclinations of the facets composing the rough interface. The illumination range depends on the distance between the substrate and the interface (Subsection 3.B).

For a short substrate-interface distance, the facets are illuminated by a Lambertian light over their full hemisphere, without any shadowing effect. The reflectance of each facet is the internal reflectance $r_{i}$ of a planar interface. The internal reflectance $\bar{r}_{i}$ of the rough interface is therefore identical to $r_{i}$, as expressed by Eq. (8).

Let us consider the case of a large substrate-interface distance. Since the illumination range is $\Omega_{\mathbf{N}}$ (Subsection 3.B), the facets are illuminated only over a part of their hemisphere. Their contribution to the reflected irradiance, and therefore to the interface's internal reflectance, depends on their orientation. For all facet orientations, we sum up the local incident and reflected irradiances and obtain the incident irradiance $E_{i}$ and the reflected irradiance $E_{r}$ of the whole rough interface. The ratio $E_{r} / E_{i}$ yields the interface's internal reflectance $\bar{r}_{i}=r_{i}^{\prime}$.

The diffusing substrate, assimilated to a Lambertian emitter of uniform radiance $L_{i}$, emits a flux element $d^{2} \Phi_{i}(\mathbf{V})$ in a direction $\mathbf{V} \in \Omega_{\mathbf{N}}$ through a solid angle $\mathrm{dV}_{\mathbf{N}}$ :

$$
d^{2} \Phi_{i}(\mathbf{V})=L_{i} d s\langle\mathbf{V} \cdot \mathbf{N}\rangle \mathrm{dV}_{\mathbf{N}} .
$$

The probability that this flux reaches the facets of normal vector $\mathbf{H} \in \boldsymbol{\Omega}_{\mathbf{N}}$ is $G_{m}(\mathbf{V}, \mathbf{H}) P(\mathbf{H})$, where the illumination probability function $G_{m}(\mathbf{V}, \mathbf{H})$ gives the proportion of the facet's illuminated area [Eq. (36)], and $P(\mathbf{H})$ gives the proportion of facets with normal vector $\mathbf{H}$ contained within the rough interface [Eq. (33)]. The flux element $d^{2} \Phi_{i}(\mathbf{V}, \mathbf{H})$ directed according to $\mathbf{V}$ received by facets of normal vector $\mathbf{H}$ is therefore

$$
d^{2} \Phi_{i}(\mathbf{V}, \mathbf{H})=G_{m}(\mathbf{V}, \mathbf{H}) P(\mathbf{H}) d^{2} \Phi_{i}(\mathbf{V}),
$$

which becomes, after Eqs. (33) and (41) are inserted,

$$
d^{2} \Phi_{i}(\mathbf{V}, \mathbf{H})=L_{i} d s G_{m}(\mathbf{V}, \mathbf{H}) D(\mathbf{H})\langle\mathbf{V} \cdot \mathbf{N}\rangle \mathrm{dV}_{\mathbf{N}} \mathrm{dH}_{\mathbf{N}} .
$$

The flux element $d^{2} \Phi_{r}(\mathbf{V}, \mathbf{H})$ reflected by the facets of normal vector $\mathbf{H}$ is the product of the incident flux with the Fresnel reflectivity $F_{1 / n_{\nu}}(\mathbf{V}, \mathbf{H})$ :

$d^{2} \Phi_{r}(\mathbf{V}, \mathbf{H})=L_{i} d s F_{1 / n_{\nu}}(\mathbf{V}, \mathbf{H}) G_{m}(\mathbf{V}, \mathbf{H}) D(\mathbf{H})\langle\mathbf{V} \cdot \mathbf{N}\rangle d V_{\mathbf{N}} d H_{\mathbf{N}}$.

By integrating $d^{2} \Phi_{i}(\mathbf{V}, \mathbf{H}) / d s$ and $d^{2} \Phi_{r}(\mathbf{V}, \mathbf{H}) / d s$ according to $d V_{\mathbf{N}}$ over $\Omega_{\mathbf{N}}$, we obtain, respectively, the irradiance incident to and the irradiance reflected by the facets of normal $\mathbf{H}$. Then, by integrating these two irradiances according to $\mathrm{dH}_{\mathbf{N}}$ over $\Omega_{\mathrm{N}}$, we obtain, respectively, the irradiance $E_{i}$ incident to and the irradiance $E_{r}$ reflected by the whole rough print-air interface. The ratio $E_{r} / E_{i}$ yields the internal reflectance $r_{i}^{\prime}$ of the rough printair interface when that interface is at a large distance from the diffusing substrate:

$$
\begin{aligned}
r_{i}^{\prime} & =\frac{E_{r}}{E_{i}} \\
& =\frac{\int_{\mathbf{H} \in \Omega_{\mathbf{N}}} \int_{\mathbf{V} \in \Omega_{\mathbf{N}}} F_{1 / n_{\nu}}(\mathbf{V}, \mathbf{H}) G_{m}(\mathbf{V}, \mathbf{H}) D(\mathbf{H})\langle\mathbf{V} \cdot \mathbf{N}\rangle \mathrm{dV}_{\mathbf{N}} \mathrm{dH}_{\mathbf{N}}}{\int_{\mathbf{H} \in \Omega_{\mathbf{N}}} \int_{\mathbf{V} \in \Omega_{\mathbf{N}}} G_{m}(\mathbf{V}, \mathbf{H}) D(\mathbf{H})\langle\mathbf{V} \cdot \mathbf{N}\rangle \mathrm{dV}_{\mathbf{N}} \mathrm{dH}_{\mathbf{N}}} .
\end{aligned}
$$

In order to facilitate the numerical computation of $r_{i}^{\prime}$, we give in Appendix B an angular variant of Eq. (43). In Table 3 below, we give numerical values showing the evolution of $r_{i}^{\prime}$ with respect to the rms slope $m$ and show the influence of shadowing.

According to the principle of conservation of energy, the transmittance $\bar{t}_{i}$ of the rough print-air interface is

$$
\bar{t}_{i}=1-\bar{r}_{i},
$$

where $\bar{r}_{i}=r_{i}$ is for short and $\bar{r}_{i}=r_{i}^{\prime}$ is for large substrateto-interface distances.

\section{Transmission of the Emerging Irradiance through the} Rough Air-Print Interface

Let us calculate the irradiance $V_{r}^{\prime}$ emerging from the print and captured by an integrating sphere. It is the part of the irradiance $W_{r}^{\prime}$ [Eq. (40)] that is transmitted through the rough interface. Since the transmittance of the printair interface is $1-\bar{r}_{i}$, the relation between the irradiance $V_{r}^{\prime}$ emerging from the print and the irradiance $W_{r}^{\prime}$ incident onto the print side of the rough print-air interface is

$$
V_{r}^{\prime}=\left(1-\bar{r}_{i}\right) W_{r}^{\prime} .
$$

The expression of $W_{r}^{\prime}$ results from the combination of Eqs. (38)-(40):

$$
W_{r}^{\prime}=\tau_{n_{\nu}}(\mathbf{L}) \cdot \frac{\rho_{B}(1-a+a t)^{2}}{1-\bar{r}_{i} \rho_{B}\left(1-a+a t^{2}\right)} V_{i}^{\prime} .
$$


By inserting Eq. (46) into Eq. (45), we obtain the complete expression of the emerging irradiance $V_{r}^{\prime}$ :

$$
V_{r}^{\prime}=\tau_{n_{\nu}}(\mathbf{L}) \cdot \frac{\rho_{B}(1-a+a t)^{2}}{1-\bar{r}_{i} \rho_{B}\left(1-a+a t^{2}\right)} \cdot\left(1-\bar{r}_{i}\right) V_{i}^{\prime} .
$$

\section{Transmission of the Radiance Emerging in the}

Direction of a Radiance Detector

In contrast to the incident irradiance $W_{r}^{\prime}$, the emerging irradiance $V_{r}^{\prime}$ is not Lambertian (see Subsection 2.A). Consequently, the radiance $L_{r}^{\prime}$ captured by a radiance detector oriented according to $\mathbf{R}$ cannot be derived directly from the emerging irradiance $V_{r}^{\prime}$. We therefore follow the same reasoning line as in Subsection 2.A by starting from the definition of radiance in order to express $L_{r}^{\prime}$ as a function of the Lambertian incident irradiance $W_{r}^{\prime}$.

Let us express $L_{r}^{\prime}$ as the flux element $d^{2} \Phi$ received by the detector along the normal $\mathbf{R}^{-}$of its surface $d s_{d}$ within its solid angle $d_{R_{R^{-}}^{-}}^{-}$(Fig. 5):

$$
L_{r}^{\prime}=\frac{d^{2} \Phi}{d s_{d} \mathrm{dR}_{\mathbf{R}^{-}}^{-}} .
$$

We call $d^{2} \Phi\left(\mathbf{R}^{-}, \mathbf{H}\right)$ the flux received by the detector from facets of normal $\mathbf{H}$, which contribute to the flux element $d^{2} \Phi$ by a probability $P(\mathbf{H})$. Since $\mathbf{R}$ was chosen to make the shadowing effect insignificant, the flux $d^{2} \Phi$ is the sum of all flux elements $d^{2} \Phi\left(\mathbf{R}^{-}, \mathbf{H}\right)$. Therefore,

$$
L_{r}^{\prime}=\frac{d^{2} \Phi}{d s_{d} \mathrm{dR}_{\mathbf{R}^{-}}^{-}}=\frac{\int_{\Omega_{\mathbf{N}}} d^{2} \Phi\left(\mathbf{R}^{-}, \mathbf{H}\right) D(\mathbf{H}) \mathrm{dH}_{\mathbf{N}}}{d s_{d} \mathrm{dR}_{\mathbf{R}^{-}}^{-}} .
$$

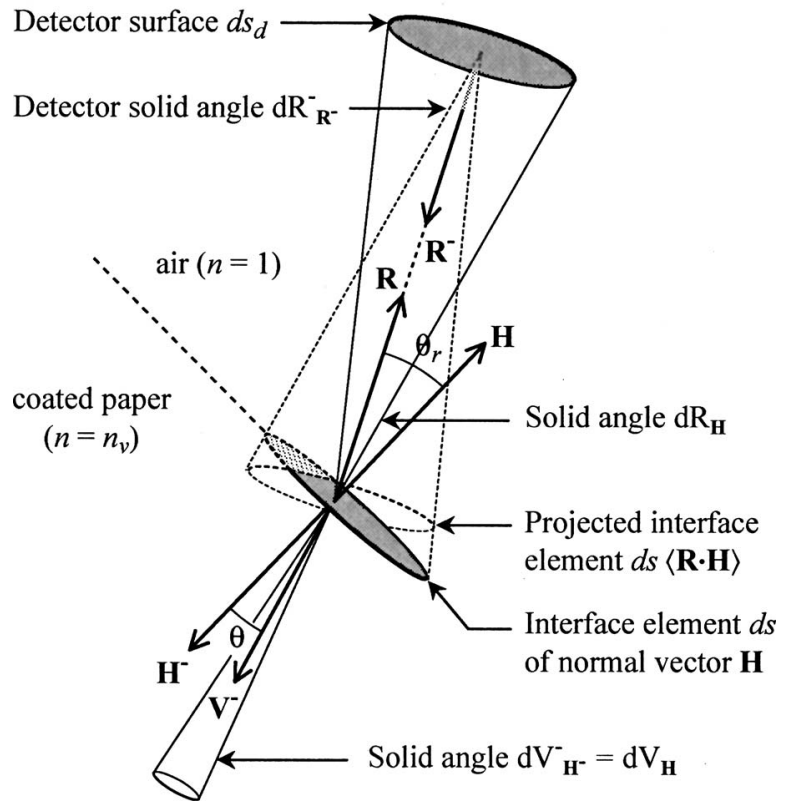

Fig. 5. Inclined facet elements $d s$ of a rough interface, of normal vector $\mathbf{H}$, receives a radiance from direction $\mathbf{V}^{-}$, within a solid angle $\mathrm{dV}_{\mathbf{H}^{-}}^{-}=\mathrm{dV}_{\mathbf{H}}=\sin \theta \mathrm{d} \theta \mathrm{d} \phi$. The radiance transmitted into the air, in direction $\mathbf{R}$ within a solid angle $\mathrm{dR}_{\mathbf{H}}=\sin \theta_{r} \mathrm{~d} \theta_{r} \mathrm{~d} \phi_{r}$, is equal to the radiance received by the surface $d s_{d}$ of the detector within its solid angle $\mathrm{dR}_{\mathbf{R}^{-}}^{-}$. Vectors $\mathbf{V}, \mathbf{H}$, and $\mathbf{R}$ are related according to Snell's refraction law.
The term $d^{2} \Phi\left(\mathbf{R}^{-}, \mathbf{H}\right) / d s_{d} \mathrm{dR}_{\mathbf{R}^{-}}^{-}$corresponds to the elementary radiance received by the detector from the facets of normal vector $\mathbf{H}$. According to the radiance invariance principle, ${ }^{20}$ this elementary radiance can also be expressed as the flux element $d^{2} \Phi(\mathbf{R}, \mathbf{H})$ emerging from the facets into direction $\mathbf{R}$ within the solid angle $d_{R_{H}}$ (Fig. 5):

$$
\frac{d^{2} \Phi\left(\mathbf{R}^{-}, \mathbf{H}\right)}{d s_{d} \mathrm{dR}_{\mathbf{R}^{-}}^{-}}=\frac{d^{2} \Phi(\mathbf{R}, \mathbf{H})}{d s\langle\mathbf{R} \cdot \mathbf{H}\rangle \mathrm{dR}_{\mathbf{H}}} .
$$

The flux element $d^{2} \Phi(\mathbf{R}, \mathbf{H})$ is the transmitted component of an incident flux element $d^{2} \Phi_{i}(\mathbf{V}, \mathbf{H})$, emitted by the diffusing substrate into a direction $\mathbf{V}$, with vectors $\mathbf{V}$, $\mathbf{H}$, and $\mathbf{R}$ being related according to Snell's refraction laws:

$$
d^{2} \Phi(\mathbf{R}, \mathbf{H})=T_{n_{\nu}}(\mathbf{R}, \mathbf{H}) d^{2} \Phi_{i}(\mathbf{V}, \mathbf{H}),
$$

where $T_{n_{\nu}}(\mathbf{R}, \mathbf{H})$ is the Fresnel transmittivity. Since the diffusing substrate is a Lambertian emitter of irradiance $W_{r}^{\prime}$, the flux $d^{2} \Phi_{i}(\mathbf{V}, \mathbf{H})$, contained in a solid angle $\mathrm{dV}_{\mathbf{H}}$, is related to the radiance $W_{r}^{\prime} / \pi$ by

$$
d^{2} \Phi_{i}(\mathbf{V}, \mathbf{H})=\frac{W_{r}^{\prime}}{\pi} d s\langle\mathbf{V} \cdot \mathbf{H}\rangle \mathrm{dV}_{\mathbf{H}} .
$$

Furthermore, by following the same reasoning line as for Eq. (19), we have

$$
\frac{\langle\mathbf{V} \cdot \mathbf{H}\rangle \mathrm{dV}_{\mathbf{H}}}{\langle\mathbf{R} \cdot \mathbf{H}\rangle \mathrm{dR}_{\mathbf{H}}}=\frac{1}{n_{v}^{2}} .
$$

With Eqs. (48)-(52), the radiance $L_{r}^{\prime}$ becomes

$$
L_{r}^{\prime}=\frac{W_{r}^{\prime}}{\pi} \frac{1}{n_{\nu}^{2}} \int_{\Omega_{\mathbf{N}}} T_{n_{\nu}}(\mathbf{R}, \mathbf{H}) D(\mathbf{H}) \mathrm{dH}_{\mathbf{H}} .
$$

In a similar manner, as the integral $\tau_{n_{\nu}}(\mathbf{L})$ was defined in Eq. (39), we define

$$
\tau_{n_{\nu}}(\mathbf{R})=\int_{\Omega_{\mathbf{N}}} T_{n_{\nu}}(\mathbf{R}, \mathbf{H}) D(\mathbf{H}) \mathrm{dH}_{\mathbf{H}} .
$$

Finally, by inserting the expression of $W_{r}^{\prime}$ [Eq. (46)] into Eq. (53), we obtain the complete expression of the radiance $L_{r}^{\prime}$ emerging from the halftone print into the direction $\mathbf{R}$ of the radiance detector:

$$
L_{r}^{\prime}=\tau_{n_{\nu}}(\mathbf{L}) \cdot \frac{\rho_{B}(1-a+a t)^{2}}{1-\bar{r}_{i} \rho_{B}\left(1-a+a t^{2}\right)} \cdot \frac{\tau_{n_{\nu}}(\mathbf{R})}{n_{\nu}^{2}} \cdot \frac{V_{i}^{\prime}}{\pi} .
$$

Expressions (47) and (55) have the same form as expressions (13) and (21) but with the Fresnel transmittivities and the internal reflectances weighted according to the facet orientations.

\section{B. Expressing the Clapper-Yule Reflectance Factor for Rough Interfaces}

We can derive from Eqs. (47) and (55) the reflectance factors of the matte halftone print for both the $\mathbf{L}$ /diffuse and the $\mathbf{L} / \mathbf{R}$ geometries. As in Section 2, we consider as the reference white the perfectly white diffuse reflector as well as the unprinted printing support. We obtain four re- 
Table 1. Main Expressions Resulting from the Classical and Extended Clapper-Yule Models ${ }^{a}$

\begin{tabular}{|c|c|c|c|}
\hline & \multirow{3}{*}{$\begin{array}{c}\text { Classical Clapper-Yule model } \\
\text { Glossy support } \\
\end{array}$} & \multirow{2}{*}{\multicolumn{2}{|c|}{ Extended Clapper-Yule model }} \\
\hline & & & \\
\hline & & Short substrate-interface distance & Large substrate-interface distance \\
\hline Transmission of the incident light & $T_{n_{v}}(\mathbf{L}, \mathbf{N})$ & (Eq. 39) & (Eq. 39) \\
\hline $\begin{array}{l}\text { Internal reflectance of the } \\
\text { print-air interface }\end{array}$ & (Eq. 8) & (Eq. 8) & $\bar{r}_{i}=r_{i}^{\prime}$ \\
\hline \multirow{2}{*}{$\begin{array}{l}\text { Attenuation due to the multiple } \\
\text { internal reflections }\end{array}$} & \multirow{2}{*}{$\frac{\rho_{B}(1-a+a t)^{2}}{1-r_{i} \rho_{B}\left(1-a+a t^{2}\right)}$} & \multirow{2}{*}{$\frac{\rho_{B}(1-a+a t)^{2}}{1-r_{i} \rho_{B}\left(1-a+a t^{2}\right)}$} & \multirow{2}{*}{$\frac{\rho_{B}(1-a+a t)^{2}}{1-r_{i}^{\prime} \rho_{B}\left(1-a+a t^{2}\right)}$} \\
\hline & & & \\
\hline \multirow{2}{*}{$\begin{array}{l}\text { Transmission of the emerging } \\
\text { light (Integrating sphere and } \\
\text { L/R geometries) }\end{array}$} & (Eq. 11) & (Eq. 44) & (Eq. 44) \\
\hline & $T_{n_{v}}(\mathbf{R}, \mathbf{N})$ & (Eq. 54) & (Eq. 54) \\
\hline \multirow{2}{*}{$\begin{array}{l}\text { Reflectance factor } \\
\text { for an integrating sphere geometry } \\
\text { and a white reflector reference }\end{array}$} & $T_{n_{v}}(\mathbf{L}, \mathbf{N})\left(1-r_{i}\right) \rho_{B}(1-a+a t)^{2}$ & \multirow{2}{*}{$\frac{\tau_{n_{v}}(\mathbf{L})\left(1-r_{i}\right) \rho_{B}(1-a+a t)^{2}}{1-r_{i} \rho_{B}\left(1-a+a t^{2}\right)}$} & \multirow{2}{*}{$\frac{\tau_{n_{v}}(\mathbf{L})\left(1-r_{i}^{\prime}\right) \rho_{B}(1-a+a t)^{2}}{1-r_{i}^{\prime} \rho_{B}\left(1-a+a t^{2}\right)}$} \\
\hline & $1-r_{i} \rho_{B}\left(1-a+a t^{2}\right)$ & & \\
\hline \multirow{2}{*}{$\begin{array}{l}\text { Reflectance factor } \\
\text { for an } \mathbf{L} / \mathbf{R} \text { geometry and a white } \\
\text { reflector reference }\end{array}$} & $T_{n_{v}}(\mathbf{L}, \mathbf{N}) T_{n_{v}}(\mathbf{R}, \mathbf{N}) \rho_{B}(1-a+a t)^{2}$ & \multirow{2}{*}{$\frac{\tau_{n_{v}}(\mathbf{L}) \tau_{n_{v}}(\mathbf{R}) \rho_{B}(1-a+a t)^{2}}{n_{v}^{2}\left(1-r_{i} \rho_{B}\left(1-a+a t^{2}\right)\right)}$} & \multirow{2}{*}{$\frac{\tau_{n_{v}}(\mathbf{L}) \tau_{n_{v}}(\mathbf{R}) \rho_{B}(1-a+a t)^{2}}{n_{v}^{2}\left(1-r_{i}^{\prime} \rho_{B}\left(1-a+a t^{2}\right)\right)}$} \\
\hline & $n_{v}^{2}\left(1-r_{i} \rho_{B}\left(1-a+a t^{2}\right)\right)$ & & \\
\hline \multirow{2}{*}{$\begin{array}{l}\text { Reflectance factor } \\
\text { for both geometries and an } \\
\text { unprinted support reference }\end{array}$} & $\left(1-r_{i} \rho_{B}\right)(1-a+a t)^{2}$ & \multirow{2}{*}{$\frac{\left(1-r_{i} \rho_{B}\right)(1-a+a t)^{2}}{1-r_{i} \rho_{B}\left(1-a+a t^{2}\right)}$} & \multirow{2}{*}{$\frac{\left(1-r_{i}^{\prime} \rho_{B}\right)(1-a+a t)^{2}}{1-r_{i}^{\prime} \rho_{B}\left(1-a+a t^{2}\right)}$} \\
\hline & $1-r_{i} \rho_{B}\left(1-a+a t^{2}\right)$ & & \\
\hline
\end{tabular}

${ }^{a}$ Shaded cells designate roughness-dependent terms.

flectance factors $R_{\Omega w}^{\prime}(\lambda), R_{\Omega p}^{\prime}(\lambda), R_{\delta w}^{\prime}(\lambda)$, and $R_{\delta p}^{\prime}(\lambda)$ for rough interfaces, which are extensions of the four reflectance factors $R_{\Omega w}(\lambda), R_{\Omega p}(\lambda), R_{\delta w}(\lambda)$, and $R_{\delta p}(\lambda)$ expressed in Eqs. (20)-(25) for planar interfaces.

\section{L/Diffuse Geometry}

The reflectance $V_{r}^{\prime} / V_{i}^{\prime}$ of the halftone print derived from Eq. (47), divided by the reflectance of a perfectly white reflector that is 1 for all wavelengths over the visible range, yields the reflectance factor $R_{\Omega w}^{\prime}$ :

$$
R_{\Omega w}^{\prime}=\tau_{n_{\nu}}(\mathbf{L})\left(1-\bar{r}_{i}\right) \frac{\rho_{B}(1-a+a t)^{2}}{1-\bar{r}_{i} \rho_{B}\left(1-a+a t^{2}\right)} .
$$

By dividing the reflectance $V_{r}^{\prime} / V_{i}^{\prime}$ of the halftone print [Eq. (47)] by the reflectance of the unprinted paper [obtained by setting $a=0$ in Eq. (47)], we express the reflectance factor $R_{\Omega p}^{\prime}$ of the halftone print by reference to the unprinted paper:

$$
R_{\Omega p}^{\prime}=\frac{\left(1-\bar{r}_{i} \rho_{B}\right)(1-a+a t)^{2}}{1-\bar{r}_{i} \rho_{B}\left(1-a+a t^{2}\right)} .
$$

The expression (57) for $R_{\Omega p}^{\prime}$ is very close to the expression (23) obtained for a planar interface. For large substrate-interface distances, $R_{\Omega p}^{\prime}$ is roughness dependent owing to the internal reflectance $\bar{r}_{i}=r_{i}^{\prime}$ of the rough interface. For short substrate-interface distances, the internal reflectance $\bar{r}_{i}=r_{i}$ is equal to the reflectance of a planar interface. In this case, $R_{\Omega p}^{\prime}$ is roughness independent and identical to the reflectance factor $R_{\Omega p}$ obtained in Eq. (23), thanks to the classical Clapper-Yule model.

\section{L/R Geometry}

The radiance detector captures the radiance $L_{r}^{\prime}$ given by Eq. (55). We obtain a reflectance factor $R_{\delta w}^{\prime}$ by dividing $L_{r}^{\prime}$ by the radiance $L_{\text {ref }}^{\prime}=V_{i}^{\prime} / \pi$ captured by the detector from a perfectly white diffuse reflector:

$$
R_{\delta w}^{\prime}=\tau_{n_{\nu}}(\mathbf{L}) \frac{\tau_{n_{\nu}}(\mathbf{R})}{n_{\nu}^{2}} \frac{\rho_{B}(1-a+a t)^{2}}{1-\bar{r}_{i} \rho_{B}\left(1-a+a t^{2}\right)} .
$$

If we choose the unprinted paper as the reference, the reflectance factor of the halftone print $R_{\delta p}^{\prime}$ is obtained by dividing $L_{r}^{\prime}$ by the radiance captured from the unprinted printing support, derived from Eq. (55) with $a=0$. As observed in Subsection 1.B for planar interfaces, $R_{\delta p}^{\prime}$ is equal to $R_{\Omega p}^{\prime}$ :

$$
R_{\delta p}^{\prime}=\frac{\left(1-\bar{r}_{i} \rho_{B}\right)(1-a+a t)^{2}}{1-\bar{r}_{i} \rho_{B}\left(1-a+a t^{2}\right)} .
$$

The internal reflectance $\bar{r}_{i}=r_{i}$ is independent of roughness when the substrate is in contact with the print-air interface.

\section{DISCUSSION}

Table 1 summarizes the main components of the classical and extended Clapper-Yule models, as well as the reflectance factors, according to the combinations of the measuring geometry and the selected reference white. Column 2 contains the expressions according to the classical Clapper-Yule model. These expressions were developed for glossy coated papers having a smooth surface. Columns 3 and 4 contain the corresponding expressions, taking into account the roughness of the print-air interface. We distinguish large and short distances between the diffusing substrate and the print-air interface.

The reflectance factors of printing supports whose interface with air is at a large distance from the diffusing substrate depend on roughness, independently of the measuring geometry or the reference white support. When the diffusing substrate is close to the interface and the reference white is a perfectly white diffuse reflector, the reflectance factor is roughness dependent only be- 
cause of the rough interface transmittances (average Fresnel transmittivities). When the reference white is the unprinted printing support, the reflectance factor becomes roughness independent.

In order to estimate the evolution of the transmittance terms, the internal reflectance terms, and the reflectance factors as a function of the print surface roughness, we have performed numerical evaluations (Tables $3-6$ below). The coating refractive index is $n_{\nu}=1.53$, as in the original paper of Williams and Clapper. ${ }^{26}$ The reflectance of the diffusing substrate is $\rho_{B}=0.9$. Hansson ${ }^{25}$ has derived from profilometric measurements the rms slope of various types of paper (Table 2). The rms slope value of coated matte paper is confirmed by confocal laser scanning microscope measurements performed by Béland and Bennett. $^{27}$

We choose four values of $m$ between 0 and 0.2 . When $m=0$, we evaluate the classical Clapper-Yule model. For other roughness values, we evaluate the extended model and give the relative deviation from the classical Clapper-Yule model.

Our extended Clapper-Yule model contains three roughness-dependent components: the average Fresnel transmittivity $\tau_{n_{\nu}}(\mathbf{L})$ of the rough interface for the incident light, the average Fresnel transmittivity $\tau_{n_{\nu}}(\mathbf{R})$ of the rough interface for the light emerging in the direction of a radiance detector, and the internal reflectance $r_{i}^{\prime}$ of the rough print-air interface at a large distance from the diffusing substrate. We consider the $45^{\circ} / 0^{\circ}$ geometry, where $\mathbf{L}$ forms an angle of $45^{\circ}$ with the print surface normal and $\mathbf{R}$ is normal to the print surface. Numerical evaluations of $\tau_{n_{\nu}}\left(45^{\circ}\right), \tau_{n_{\nu}}\left(0^{\circ}\right)$, and $r_{i}^{\prime}$ as functions of roughness are presented in Table 3.

Table 3 shows that, for the range of roughness levels over which the evaluations are performed, the deviation of the evaluated terms with respect to a planar interface is small. The average Fresnel transmittivity $\tau_{n_{\nu}}\left(0^{\circ}\right)$ for an incidence angle of $0^{\circ}$ is constant. The deviation of the average Fresnel transmittivity $\tau_{n}\left(45^{\circ}\right)$ for an incidence angle of $45^{\circ}$ is less than $0.5 \%$. To show the influence of the shadowing effect, we first calculate the internal reflectance $r_{i}^{\prime}$ according to Eq. (43), where shadowing is taken into account, and then calculated it by ignoring shadowing, i.e., setting $G_{m}=1$ in Eq. (43). For a close distance to the diffusing substrate, the terms $\tau_{n_{\nu}}\left(45^{\circ}\right)$ and $\tau_{n_{\nu}}\left(0^{\circ}\right)$ are identical to the ones for a large distance. The deviation of the internal reflectance $r_{i}^{\prime}$ of a print-air interface far from the diffusing substrate is less than $0.6 \%$. Let us see how these terms influence the reflectance factors.

Table 2. Rms Slopes of Various Paper Types ${ }^{a}$

\begin{tabular}{lc}
\hline Type of Paper & Rms Slope $m$ \\
\hline Cast-coated paper & 0.025 \\
Copy paper & 0.193 \\
Heavy-coated glossy paper & 0.034 \\
Heavy-coated matte paper & 0.046 \\
Lightweight coated paper & 0.069 \\
Newsprint paper & 0.185 \\
Supercalendered paper & 0.075 \\
\hline
\end{tabular}

${ }^{a}$ Measurements performed by Hansson. ${ }^{25}$
Table 3. Evaluation of Roughness-Dependent Terms for Various Roughnesses (rms Slope $m)^{a}$

Average Fresnel Transmittivities and Internal Reflectances

\begin{tabular}{lcccc} 
& 0 & 0.05 & 0.1 & 0.2 \\
\cline { 2 - 5 } arctan $m$ & $0^{\circ}$ & $2.9^{\circ}$ & $5.7^{\circ}$ & $11.3^{\circ}$ \\
\hline$\tau_{n_{\nu}}\left(45^{\circ}\right)$ & 0.946 & 0.945 & 0.943 & 0.935 \\
& & $\sim 0 \%$ & $0.1 \%$ & $0.5 \%$ \\
$\tau_{n_{\nu}}\left(0^{\circ}\right)$ & 0.956 & 0.956 & 0.956 & 0.956 \\
$r_{i}^{\prime}$ & & $\sim 0 \%$ & $\sim 0 \%$ & $\sim 0 \%$ \\
$($ with shadowing) & 0.614 & 0.614 & 0.615 & 0.618 \\
$r_{i}^{\prime}$ & & $0.1 \%$ & $0.2 \%$ & $0.6 \%$ \\
(no shadowing) & 0.614 & 0.615 & 0.616 & 0.621 \\
\hline
\end{tabular}

${ }^{a}$ Average Fresnel transmittivity $\tau_{n}\left(45^{\circ}\right)$ of the rough print-air interface for collimated light incident at $45^{\circ}$, average Fresnel transmittivity $\tau_{n_{v}}\left(0^{\circ}\right)$ of the rough interface for emerging light at $0^{\circ}$, and internal reflectance $r_{i}^{\prime}$ of the rough interface at a large distance from the diffusing substrate.

Table 4. Deviations of Reflectance Factor $R_{\Omega w}^{\prime} a$

\begin{tabular}{|c|c|c|c|c|}
\hline rms Slope $m$ & 0 & 0.05 & 0.1 & 0.2 \\
\hline \multicolumn{5}{|l|}{$t=0.9$} \\
\hline$a=0.25$ & 0.659 & $\begin{array}{c}0.659 \\
0.1 \%\end{array}$ & $\begin{array}{l}0.657 \\
0.3 \%\end{array}$ & $\begin{array}{l}0.650 \\
1.4 \%\end{array}$ \\
\hline$a=0.5$ & 0.593 & $\begin{array}{c}0.592 \\
0.1 \%\end{array}$ & $\begin{array}{l}0.591 \\
0.4 \%\end{array}$ & $\begin{array}{l}0.584 \\
1.5 \%\end{array}$ \\
\hline$a=0.75$ & 0.534 & $\begin{array}{c}0.534 \\
0.1 \%\end{array}$ & $\begin{array}{l}0.532 \\
0.4 \%\end{array}$ & $\begin{array}{l}0.526 \\
1.6 \%\end{array}$ \\
\hline \multicolumn{5}{|l|}{$t=0.5$} \\
\hline$a=0.25$ & 0.456 & $\begin{array}{l}0.456 \\
0.1 \%\end{array}$ & $\begin{array}{l}0.455 \\
0.4 \%\end{array}$ & $\begin{array}{l}0.449 \\
1.6 \%\end{array}$ \\
\hline$a=0.5$ & 0.282 & $\begin{array}{l}0.282 \\
0.2 \%\end{array}$ & $\begin{array}{l}0.281 \\
0.5 \%\end{array}$ & $\begin{array}{l}0.277 \\
1.9 \%\end{array}$ \\
\hline$a=0.75$ & 0.169 & $\begin{array}{c}0.169 \\
0.2 \%\end{array}$ & $\begin{array}{c}0.168 \\
0.6 \%\end{array}$ & $\begin{array}{l}0.166 \\
1.9 \%\end{array}$ \\
\hline \multicolumn{5}{|l|}{$t=0.1$} \\
\hline$a=0.25$ & 0.338 & $\begin{array}{l}0.337 \\
0.1 \%\end{array}$ & $\begin{array}{l}0.336 \\
0.4 \%\end{array}$ & $\begin{array}{l}0.333 \\
1.7 \%\end{array}$ \\
\hline$a=0.5$ & 0.138 & $\begin{array}{l}0.138 \\
0.2 \%\end{array}$ & $\begin{array}{l}0.137 \\
0.5 \%\end{array}$ & $\begin{array}{l}0.135 \\
1.9 \%\end{array}$ \\
\hline$a=0.75$ & 0.040 & $\begin{array}{l}0.040 \\
0.2 \%\end{array}$ & $\begin{array}{l}0.040 \\
0.5 \%\end{array}$ & $\begin{array}{c}0.040 \\
2 \%\end{array}$ \\
\hline
\end{tabular}

${ }^{a}$ Reflectance factor $R_{\Omega_{w}}^{\prime}$ of a printing support coated with a thick transparent layer defined for an integrating-sphere measuring geometry in reference to a white diffuse reflector, for various roughnesses $m$, ink transmittances, $t$, and relative ink coverages $a$. At $m=0$, the reflectance factor is the one of the classical Clapper-Yule model.

Tables 4-6 show the corresponding deviations of the reflection factors, for an interface that is far from the diffusing substrate. Table 4 shows the reflectance factor $R_{\Omega w}^{\prime}$ for a $0 \%$ diffuse geometry and a perfectly white diffuse reflector reference, Table 5 shows the reflectance factor $R_{\delta w}^{\prime}$ for a $45^{\circ} / 0^{\circ}$ geometry and a perfectly white diffuse reflector reference, and Table 6 shows the reflectance factor $R_{p}^{\prime}$ with the unprinted printing support as reference white (same expression for a $\mathbf{L} /$ diffuse geometry as for a $\mathbf{L} / \mathbf{R}$ geometry).

The reflectance factors yielded by our extended model and by the classical Clapper-Yule model are nearly iden- 
tical for rms slopes lower than 0.05 (maximum deviation of $0.2 \%$ ). Therefore, with respect to the Clapper-Yule model, a print-air interface that is far from the diffusing substrate and whose rms slope is lower than 0.05 can be considered flat. For rougher print surfaces, the evolution of the reflectance factor depends mainly on the type of reference white support and to a smaller extent on the measuring geometry.

When the reference white support is a perfectly white diffuse reflector, the $45 \%$ diffuse geometry yields reflectance factor deviations up to $0.6 \%$ for $m=0.1$ and up to $2 \%$ for $m=0.2$ (reflectance factor $R_{\Omega w}$, Table 4 ). The $45^{\circ} / 0^{\circ}$ geometry yields deviations up to $0.2 \%$ for $m=0.1$ and up to $1 \%$ for $m=0.2$ (reflectance factor $R_{\delta w}$, Table 5 ). These deviations are principally due to the average Fresnel transmittivity for the incident light $\tau_{n_{\nu}}\left(45^{\circ}\right)$ and the average Fresnel transmittivity for the emerging light (1 $\left.-r_{i}^{\prime}\right)$ or $\tau_{\nu_{n}}\left(0^{\circ}\right) / n_{\nu}^{2}$, respectively. Since $\tau_{\nu_{n}}\left(0^{\circ}\right)$ is almost roughness independent, the $45^{\circ} / 0^{\circ}$ geometry yields smaller deviations than the $45 \%$ diffuse geometry does.

When the reference white support is the unprinted printing support, the reflectance factor $R_{p}^{\prime}$ does not contain any Fresnel transmittivity term and depends on the roughness level only because of to the print-air interface internal reflectance $r_{i}^{\prime}$. As a consequence, the evolution of the reflection factor $R_{p}^{\prime}$ with respect to the surface roughness is very small (maximum deviation of $0.6 \%$ between reflectance factors calculated according to the extended Clapper-Yule model for a rms slope $m=0.2$ and according to the classical Clapper-Yule model for $m=0$ ).

The reflectance factors of printing supports whose diffusing substrate is close to the print-air interface have

\section{Table 5. Deviations of Reflectance Factor $\boldsymbol{R}_{\delta w}^{\prime}{ }^{a}$}

\begin{tabular}{|c|c|c|c|c|}
\hline rms Slope $m$ & 0 & 0.05 & 0.1 & 0.2 \\
\hline \multicolumn{5}{|l|}{$t=0.9$} \\
\hline$a=0.25$ & 0.697 & $\begin{array}{c}0.697 \\
\sim 0 \%\end{array}$ & $\begin{array}{c}0.697 \\
\sim 0 \%\end{array}$ & $\begin{array}{l}0.694 \\
0.5 \%\end{array}$ \\
\hline$a=0.5$ & 0.627 & $\begin{array}{c}0.628 \\
\sim 0 \%\end{array}$ & $\begin{array}{c}0.627 \\
0.1 \%\end{array}$ & $\begin{array}{l}0.524 \\
0.5 \%\end{array}$ \\
\hline$a=0.75$ & 0.565 & $\begin{array}{c}0.565 \\
\sim 0 \%\end{array}$ & $\begin{array}{c}0.565 \\
0.1 \%\end{array}$ & $\begin{array}{l}0.562 \\
0.6 \%\end{array}$ \\
\hline \multicolumn{5}{|l|}{$t=0.5$} \\
\hline$a=0.25$ & 0.483 & $\begin{array}{l}0.483 \\
\sim 0 \%\end{array}$ & $\begin{array}{c}0.482 \\
0.1 \%\end{array}$ & $\begin{array}{l}0.462 \\
0.6 \%\end{array}$ \\
\hline$a=0.5$ & 0.299 & $\begin{array}{c}0.299 \\
\sim 0 \%\end{array}$ & $\begin{array}{l}0.298 \\
0.1 \%\end{array}$ & $\begin{array}{l}0.296 \\
0.8 \%\end{array}$ \\
\hline$a=0.75$ & 0.179 & $\begin{array}{c}0.179 \\
0 \%\end{array}$ & $\begin{array}{c}0.179 \\
0.2 \%\end{array}$ & $\begin{array}{c}0.177 \\
1 \%\end{array}$ \\
\hline \multicolumn{5}{|l|}{$t=0.1$} \\
\hline$a=0.25$ & 0.357 & $\begin{array}{l}0.357 \\
\sim 0 \%\end{array}$ & $\begin{array}{l}0.357 \\
0.1 \%\end{array}$ & $\begin{array}{l}0.355 \\
0.7 \%\end{array}$ \\
\hline$a=0.5$ & 0.146 & $\begin{array}{c}0.146 \\
\sim 0 \%\end{array}$ & $\begin{array}{c}0.146 \\
0.2 \%\end{array}$ & $\begin{array}{l}0.145 \\
0.9 \%\end{array}$ \\
\hline$a=0.75$ & 0.043 & $\begin{array}{c}0.043 \\
\sim 0 \%\end{array}$ & $\begin{array}{c}0.043 \\
0.2 \%\end{array}$ & $\begin{array}{c}0.042 \\
1 \%\end{array}$ \\
\hline
\end{tabular}

${ }^{a}$ Reflectance factor $R_{\delta w}^{\prime}$ of a printing support coated with a thick transparent layer defined for a $45^{\circ} / 0^{\circ}$ measuring geometry in reference to a white diffuse reflector, for various roughnesses $m$, ink transmittances $t$, and relative ink coverages $a$. At $m=0$, the reflectance factor is the one of the classical Clapper-Yule model.
Table 6. Deviations of Reflectance Factor

\begin{tabular}{|c|c|c|c|c|}
\hline rms Slope $m$ & 0 & 0.05 & 0.1 & 0.2 \\
\hline \multicolumn{5}{|l|}{$t=0.9$} \\
\hline$a=0.25$ & 0.898 & $\begin{array}{c}0.898 \\
\sim 0 \%\end{array}$ & $\begin{array}{c}0.898 \\
\sim 0 \%\end{array}$ & $\begin{array}{l}0.897 \\
0.1 \%\end{array}$ \\
\hline$a=0.5$ & 0.808 & $\begin{array}{l}0.808 \\
\sim 0 \%\end{array}$ & $\begin{array}{c}0.808 \\
\sim 0 \%\end{array}$ & $\begin{array}{l}0.807 \\
0.1 \%\end{array}$ \\
\hline$a=0.75$ & 0.728 & $\begin{array}{c}0.728 \\
\sim 0 \%\end{array}$ & $\begin{array}{c}0.727 \\
0.1 \%\end{array}$ & $\begin{array}{l}0.727 \\
0.2 \%\end{array}$ \\
\hline \multicolumn{5}{|l|}{$t=0.5$} \\
\hline$a=0.25$ & 0.622 & $\begin{array}{c}0.622 \\
\sim 0 \%\end{array}$ & $\begin{array}{l}0.621 \\
0.1 \%\end{array}$ & $\begin{array}{l}0.620 \\
0.2 \%\end{array}$ \\
\hline$a=0.5$ & 0.384 & $\begin{array}{c}0.384 \\
0.1 \%\end{array}$ & $\begin{array}{l}0.384 \\
0.1 \%\end{array}$ & $\begin{array}{l}0.383 \\
0.4 \%\end{array}$ \\
\hline$a=0.75$ & 0.231 & $\begin{array}{l}0.230 \\
0.1 \%\end{array}$ & $\begin{array}{c}0.230 \\
0.2 \%\end{array}$ & $\begin{array}{l}0.229 \\
0.6 \%\end{array}$ \\
\hline \multicolumn{5}{|l|}{$t=0.1$} \\
\hline$a=0.25$ & 0.460 & $\begin{array}{l}0.460 \\
\sim 0 \%\end{array}$ & $\begin{array}{l}0.460 \\
0.1 \%\end{array}$ & $\begin{array}{l}0.459 \\
0.3 \%\end{array}$ \\
\hline$a=0.5$ & 0.188 & $\begin{array}{l}0.188 \\
\sim 0 \%\end{array}$ & $\begin{array}{l}0.187 \\
0.2 \%\end{array}$ & $\begin{array}{l}0.187 \\
0.5 \%\end{array}$ \\
\hline$a=0.75$ & 0.055 & $\begin{array}{l}0.055 \\
0.1 \%\end{array}$ & $\begin{array}{l}0.055 \\
0.2 \%\end{array}$ & $\begin{array}{l}0.055 \\
0.6 \%\end{array}$ \\
\hline
\end{tabular}

${ }^{a}$ Reflectance factor $R_{p}^{\prime}$ of a printing support coated with a thick transparent layer in reference to the unprinted printing support, for various roughnesses $m$, ink transmittances $t$, and relative ink coverages $a$. At $m=0$, the reflectance factor is the one of the classical Clapper-Yule model.

not been evaluated numerically, since the internal reflectance of their print-air interface is equal to that of a planar interface and therefore roughness independent. The evolution of the reflectance factor depends only on the selected reference white.

When the reference white support is a perfectly white diffuse reflector, both the reflectance factors $R_{\Omega w}^{\prime}$ and $R_{\delta w}^{\prime}$ have a roughness-independent average Fresnel transmittivity for the emerging light [respectively, $\left(1-r_{i}\right)$ and $\tau_{\nu_{n}}\left(0^{\circ}\right) / n_{\nu}^{2}$. Therefore, they vary with the roughness level only because of the average Fresnel transmittivities for the incident light $\tau_{n_{\nu}}\left(45^{\circ}\right)$. However, the deviation of $\tau_{n_{\nu}}\left(45^{\circ}\right)$ is insignificant for rms slopes up to 0.1. Therefore, with respect to the Clapper-Yule model, a print-air interface that is close to the diffusing substrate and whose rms slope is lower than 0.1 can be considered flat.

When the reference white support is the unprinted printing support, the reflectance factor $R_{p}^{\prime}$ is roughness independent, independently of the measuring geometry, since it does not contain any Fresnel transmittivity. Hence this reflection factor can be predicted directly, thanks to the classical Clapper-Yule model.

\section{CONCLUSIONS}

We extend the Clapper-Yule model in order to include the effects of surface roughness in the prediction of halftoneprint spectral reflectance factors. The rough air-print interface is modeled by a set of randomly oriented microfacets whose slopes follow a Gaussian distribution. We apply the laws of geometrical optics to each microfacet and ob- 
tain, by integrating over all facet orientations, an average expression for the terms determining the interaction of light with the rough air-print interface. We are able to express the average Fresnel transmittivity of the rough interface for an incident collimated light of a given orientation, the internal reflectance of the rough interface, and the average Fresnel transmittivity of the rough interface for the emerging light. The expressions for the ClapperYule model for smooth or rough surfaces differ only by the value of the corresponding average Fresnel transmittivity or reflectivity terms.

When the diffusing substrate is far from the rough print-air interface (e.g., a thick nondiffusing coating), for rms facet slopes of 0.2 and in reference to a perfectly white diffuse reflector, the reflectance factors vary by $2 \%$ for an integrated sphere measuring geometry and by $1 \%$ for a $45^{\circ} / 0^{\circ}$ geometry. For both geometries, in reference to the unprinted printing support the reflection factor variations are negligible (variations of less than $0.6 \%$ ). At rms slopes of 0.1 , for all measuring geometries and white references, the reflectance factor variations are also negligible (less than $0.5 \%$ ).

When the diffusing substrate is close to the rough print-air interface (e.g., calendered paper, paper with a white diffusing coating, copy paper, or newsprint paper), the internal reflectance of the rough interface equals the internal reflectance of a planar interface and only the average Fresnel transmittivity terms for the incident and emerging light differ. They yield, however, negligible reflectance factor variations (below 0.5\%). Furthermore, when reflection factors are expressed with the unprinted paper printing support as the reference white, the average Fresnel transmittivity terms do not appear in the corresponding expressions. Reflection factors are therefore identical for smooth and for rough interfaces.

High-quality papers such as coated and calendered papers have a rms slope of less than 0.1 (see Table 2). Therefore, the influence of roughness on the reflectance factors of high-quality papers is negligible (less than $0.5 \%$ ), independently of the distance between the interface and the diffusing substrate, the measuring geometry, and the selected reference white. Uncoated low-quality papers with a rough interface, such as newsprint and photocopy papers, have a diffusing substrate close to the print-air interface and therefore also negligible reflection factor variations.

We may therefore conclude that, for the large majority of papers, rough interfaces induce only negligible variations of reflection factors. The Clapper-Yule model is therefore suitable for predicting equally well the reflection factors of both rough and glossy halftone prints.

\section{APPENDIX A: NOTATION}

Snell's laws and Fresnel's formula are generally expressed as functions of angles. However, since we introduce a large number of orientations, we prefer to use unit vectors. This simplifies considerably the formulation of equations, since a unit vector is equivalent to two angles (its spherical coordinates). Furthermore, the unit vector notation is referential independent. The spherical coordinates $(\theta, \phi)$ of a unit vector $\mathbf{V}$ are defined with respect to a reference unit vector $\mathbf{N}$. The $\operatorname{dot} \operatorname{product}\langle\mathbf{V} \cdot \mathbf{N}\rangle$ therefore denotes $\cos \theta$. A small variation of $\mathbf{V}$ around its initial position forms an elementary solid angle $\mathrm{dV}_{\mathbf{N}}=|\sin \theta| \mathrm{d} \theta \mathrm{d} \phi$ (since a solid angle is always positive, we take the absolute value of $\sin \theta$ for $\theta>\pi / 2$ ). The subscript $\mathbf{N}$ in the notation $d V_{N}$ specifies the unit vector according to which the angles are defined. This notation permits conversion of the vector notation into angular notation without any confusion in angles. A hemisphere whose basis is the plane of normal vector $\mathbf{N}$ is noted as $\Omega_{\mathbf{N}}$. It comprises all vectors for which $\langle\mathbf{V} \cdot \mathbf{N}\rangle>0$. Vector $\mathbf{V}^{-}$defines the same vector as $\mathbf{V}$ but in the opposite direction.

When a light ray oriented according to a vector $\mathbf{V}$ reaches an air-print interface (relative index of refraction $n_{\nu}$ ) of normal vector $\mathbf{N}$, the Fresnel reflectivity and transmittivity are noted, respectively, as $F_{n_{\nu}}(\mathbf{V}, \mathbf{N})$ and $T_{n_{\nu}}(\mathbf{V}, \mathbf{N})$. When the light ray reaches from below the print-air interface, the relative index of refraction is $1 / n_{\nu}$, and the corresponding Fresnel reflectivity and transmittivity terms are $F_{1 / n_{\nu}}(\mathbf{V}, \mathbf{N})$ and $T_{1 / n_{\nu}}(\mathbf{V}, \mathbf{N})$.

\section{APPENDIX B: CALCULATION OF THE ROUGH INTERFACE'S INTERNAL REFLECTANCE}

The internal reflectance of a rough interface at a large distance from the diffusing substrate is expressed in Eq. (43) as the ratio of the reflected irradiance

$$
\begin{aligned}
E_{r}= & \int_{\mathbf{V} \in \Omega_{\mathbf{N}}} \int_{\mathbf{H} \in \Omega_{\mathbf{N}}} F_{1 / n_{\nu}}(\mathbf{V}, \mathbf{H}) G_{m}(\mathbf{V}, \mathbf{H}) D(\mathbf{H}) \\
& \times\langle\mathbf{V} \cdot \mathbf{N}\rangle \mathrm{dV}_{\mathbf{N}} \mathrm{dH}_{\mathbf{N}}
\end{aligned}
$$

to the incident irradiance

$$
E_{i}=\int_{\mathbf{V} \in \Omega_{\mathbf{N}}} \int_{\mathbf{H} \in \Omega_{\mathbf{N}}} G_{m}(\mathbf{V}, \mathbf{H}) D(\mathbf{H})\langle\mathbf{V} \cdot \mathbf{N}\rangle \mathrm{dV}_{\mathbf{N}} \mathrm{dH}_{\mathbf{N}} .
$$

Let us express irradiances $E_{r}$ and $E_{i}$ as a function of angles. Further simplifications will be possible thanks to the roughness isotropy and to symmetry considerations.

The light direction vector $\mathbf{V}$ is defined by its spherical angles $(\theta, \phi)$, and the facets' normal vector $\mathbf{H}$ is defined by its spherical angles $\left(\theta_{h}, \phi_{h}\right)$. The polar angles $\theta$ and $\theta_{h}$ are defined with respect to the mean surface normal vector $\mathbf{N}$. The azimuthal angles $\phi$ and $\phi_{h}$ are defined with respect to the same arbitrary azimuthal direction. According to these definitions, we have

$$
\begin{aligned}
\langle\mathbf{V} \cdot \mathbf{N}\rangle & =\cos \theta, \\
\mathrm{dV}_{\mathbf{N}} & =|\sin \theta| \mathrm{d} \theta \mathrm{d} \phi, \\
\mathrm{dH}_{\mathbf{N}} & =\left|\sin \theta_{h}\right| \mathrm{d} \theta_{h} \mathrm{~d} \phi_{h} .
\end{aligned}
$$

The illumination probability function $G_{m}(\mathbf{V}, \mathbf{H})$, expressed in Eq. (36), is

$$
G_{m}(\mathbf{V}, \mathbf{H})=\frac{h(\langle\mathbf{V} \cdot \mathbf{H}\rangle)}{\Lambda_{m}(\theta)+1},
$$




$$
\Lambda_{m}(\theta)=\frac{1}{2}\left[\frac{1}{\sqrt{\pi}} \frac{\sqrt{2} m}{\cot \theta} \exp \left(-\frac{\cot ^{2} \theta}{2 m^{2}}\right)-\operatorname{erfc}\left(\frac{\cot \theta}{\sqrt{2} m}\right)\right] .
$$

The unit step function $h(\langle\mathbf{V} \cdot \mathbf{H}\rangle)$ is 0 when $\langle\mathbf{V} \cdot \mathbf{H}\rangle$ is negative and 1 when $\langle\mathbf{V} \cdot \mathbf{H}\rangle$ is positive, with

$$
\langle\mathbf{V} \cdot \mathbf{H}\rangle=\cos \theta \cos \theta_{h}+\sin \theta \sin \theta_{h} \cos \left(\phi-\phi_{h}\right) .
$$

The facet orientation distribution function $D(\mathbf{H})$, expressed in Eq. (34), is

$$
D(\mathbf{H})=\frac{\exp \left(-\tan ^{2} \theta_{h} / 2 m^{2}\right)}{2 \pi m^{2} \cos ^{3} \theta_{h}} .
$$

Thanks to expressions (B1)-(B3), the angular expression of $E_{r}$ is

$$
\begin{aligned}
E_{r}= & \frac{1}{2 \pi m^{2}} \int_{\phi_{h}=0}^{2 \pi} \int_{\theta_{h}=0}^{\pi / 2} \int_{\phi=0}^{2 \pi} \int_{\theta=0}^{\pi / 2} F_{1 / n_{\nu}}\left(\theta_{v h}\right) \frac{U\left(\cos \theta_{v h}\right)}{\Lambda_{m}(\theta)+1} \\
& \times \frac{\exp \left(-\tan ^{2} \theta_{h} / 2 m^{2}\right)}{\cos ^{3} \theta_{h}} \cos \theta\left|\sin \theta \| \sin \theta_{h}\right| \mathrm{d} \theta \mathrm{d} \phi \mathrm{d} \theta_{h} \mathrm{~d} \phi_{h} .
\end{aligned}
$$

Two simplifications can be performed on this quadruple integral. First, owing to the interface roughness isotropy, the integrated terms are independent of the facet orientation azimuthal angle $\phi_{h}$. The integral over $\phi_{h}$ therefore yields a factor of $2 \pi$. The single integral over the polar angle $\theta_{h}$ is sufficient to take into account all facet orientations. Let us consider that all facet normal vectors belong to the same vertical plane $\left(\phi_{h}=0\right)$, which is considered the azimuth origin. The term $\cos \theta_{\nu h}$ becomes

$$
\cos \theta_{v h}=\cos \theta \cos \theta_{h}+\sin \theta \sin \theta_{h} \cos \phi,
$$

and the azimuthal angle $\phi_{h}$ has completely disappeared from the integrand. The reflected irradiance becomes

$$
\begin{aligned}
E_{r}= & \frac{1}{m^{2}} \int_{\theta_{h}=0}^{\pi / 2} \int_{\phi=0}^{2 \pi} \int_{\theta=0}^{\pi / 2} F_{1 / n_{\nu}}\left(\theta_{v h}\right) \frac{U\left(\cos \theta_{v h}\right)}{\Lambda_{m}(\theta)+1} \\
& \times \frac{\exp \left(-\tan ^{2} \theta_{h} / 2 m^{2}\right)}{\cos ^{3} \theta_{h}} \cos \theta|\sin \theta|\left|\sin \theta_{h}\right| \mathrm{d} \theta \mathrm{d} \phi \mathrm{d} \theta_{h} .
\end{aligned}
$$

Second, the only term depending on $\phi$ in the integrand is $\cos \theta_{\nu h}$. However, $\cos \theta_{\nu h}$ is an even function according to $\phi$; i.e., it remains identical if we replace $\phi$ with $-\phi$ or $2 \pi-\phi$. Therefore, an integration from $\phi=0$ to $\pi$ is sufficient to take into account all directions of the incident diffuse light, yielding a factor of 2 :

$$
\begin{aligned}
E_{r}= & \frac{2}{m^{2}} \int_{\theta_{h}=0}^{\pi / 2} \int_{\phi=0}^{\pi} \int_{\theta=0}^{\pi / 2} F_{1 / n_{\nu}}\left(\theta_{v h}\right) \frac{U\left(\cos \theta_{v h}\right)}{\Lambda_{m}(\theta)+1} \\
& \times \frac{\exp \left(-\tan ^{2} \theta_{h} / 2 m^{2}\right)}{\cos ^{3} \theta_{h}} \cos \theta|\sin \theta|\left|\sin \theta_{h}\right| \mathrm{d} \theta \mathrm{d} \phi \mathrm{d} \theta_{h} .
\end{aligned}
$$

In expression (B5), integrals do not have an analytical solution. They are therefore calculated by a discrete summation:

$$
\begin{aligned}
E_{r}= & \frac{2}{m^{2}} \sum_{\theta_{h}=0}^{\pi / 2} \sum_{\phi=0}^{\pi} \sum_{\theta=0}^{\pi / 2} F_{1 / n_{\nu}}\left(\theta_{v h}\right) \frac{U\left(\cos \theta_{v h}\right)}{\Lambda_{m}(\theta)+1} \\
& \times \frac{\exp \left(-\tan ^{2} \theta_{h} / 2 m^{2}\right)}{\cos ^{3} \theta_{h}} \cos \theta|\sin \theta|\left|\sin \theta_{h}\right| \Delta \theta \Delta \phi \Delta \theta_{h} .
\end{aligned}
$$

The same simplifications apply to the incident irradiance $E_{i}$, which takes the same expression as Eq. (B6) but without the Fresnel function $F_{1 / n}\left(\theta_{v h}\right)$. In the final expression of $r_{i}^{\prime}=E_{r} / E_{i}$, the coefficients $2 / \mathrm{m}^{2}$ cancel each other.

Corresponding author Mathieu Hébert can be reached by e-mail at mathieu.hebert@epfl.ch.

\section{REFERENCES}

1. J. Yule and W. Nielsen, "The penetration of light into paper and its effect on halftone reproductions," in Proceedings of the Technical Association of the Graphic Arts (TAGA) Conference, 1951, pp. 65-76. http://www.taga.org.

2. J. A. S. Viggiano, "Modeling the color of multi-color halftones," in Proceedings of the Technical Association of the Graphic Arts (TAGA) Conference, 1990, pp. 44-62. http://www.taga.org.

3. R. Balasubramanian, "Optimization of the spectral Neugebauer model for printer characterization," J. Electron. Imaging 8, 156-166 (1999).

4. K. Iino and R. S. Berns, "Building color management modules using linear optimization I. Desktop," J. Imaging Sci. Technol. 41, 79-94 (1998).

5. K. Iino and R. S. Berns, "Building color management modules using linear optimization II. Prepress system for offset printing," J. Imaging Sci. Technol. 42, 99-114 (1998)

6. F. Ruckdeschel and O. Hauser, "Yule-Nielsen in printing: a physical analysis," Appl. Opt. 17, 3376-3383 (1978).

7. J. Arney, "A probability description of the Yule-Nielsen effect I," J. Imaging Sci. Technol. 41, 633-636 (1997).

8. G. Rogers, "Optical dot gain: lateral scattering probabilities," J. Imaging Sci. Technol. 42, 341-345 (1998).

9. L. Yang, R. Lenz, and B. Kruse, "Light scattering and ink penetration effects on tone reproduction," J. Opt. Soc. Am. A 18, 360-366 (2001).

10. F. Clapper and J. Yule, "The effect of multiple internal reflections on the densities of halftone prints on paper," $\mathrm{J}$. Opt. Soc. Am. 43, 600-603 (1953).

11. G. Rogers, "A generalized Clapper-Yule model of halftone reflectance," Color Res. Appl. 25, 402-407 (2000).

12. P. Emmel and R. D. Hersch. "A unified model for color prediction of halftoned prints," J. Imaging Sci. Technol. 44, 351-359 (2000).

13. R. D. Hersch, F. Collaud, F. Crété, and P. Emmel, "Spectral prediction and dot surface estimation models for halftone prints," in Color Imaging IX: Processing, Hardcopy, and Applications, R. Eschbach and G. G. Marcu; eds., Proc. SPIE 5293, 356-369 (2004).

14. J. A. Sanchez-Gil and M. Nieto-Vesperinas, "Light scattering from random rough dielectric surfaces," J. Opt. Soc. Am. A 8, 1270-1286 (1991).

15. J. Caron, J. Lafait, and C. Andraud, "Scalar Kirchhoff's model for light scattering from dielectric random rough surfaces," Opt. Commun. 207, 17-28 (2002).

16. T. Germer, "Polarized light diffusely scattered under smooth and rough interfaces," Polarization Science and Remote Sensing, J. A. Shaw and J. S. Tyo, eds., Proc. SPIE 5158, 193-204 (2003).

17. S. K. Nayar, K. Ikeuchi, and T. Kanade, "Surface reflection: physical and geometrical perspectives," IEEE Trans. Pattern Anal. Mach. Intell. 13, 611-634 (1991).

18. M. Born and E. Wolf, Principle of Optics, 7th expanded ed. (Pergamon, 1999), p. 47. 
19. D. B. Judd, "Fresnel reflection of diffusely incident light," J. Res. Natl. Bur. Stand. 29, 329-332 (1942).

20. W. R. McCluney, Introduction to Radiometry and Photometry (Artech House, 1994) pp. 7-13.

21. K. E. Torrance and E. M. Sparrow, "Theory of off-specular reflection from roughened surfaces," J. Opt. Soc. Am. 57, 1104-1114 (1967).

22. D. Becker and K. Kasper, "Digital prints: technology, materials, image quality \& stability," http:// www.foto.unibas.ch/ rundbrief/les33.htm.

23. B. G. Smith, "Geometrical shadowing of a random rough surface," IEEE Trans. Antennas Propag. AP-15, 668-671 (1967).

24. N. C. Bruce, "On the validity of the inclusion of geometrical shadowing functions in the multiple-scatter Kirchhoff approximation," Waves Random Media 14, 1-12 (2004).

25. P. Hansson, "Geometrical modeling of light scattering from paper substrates," Diploma work (Department of Engineering Sciences, The Ångström Laboratory, Uppsala University, Uppsala, Sweden, 2003). http:// www.angstrom.uu.se/solidstatephysics/dissertation_ram/ peter_hansson_art/Paper9_v15pdf.

26. F. C. Williams and F. R. Clapper, "Multiple internal reflections in photographic color prints," J. Opt. Soc. Am. 43, 595-599 (1953).

27. M. C. Béland and J. M. Bennett, "Effect of local microroughness on the gloss uniformity of printed paper surfaces," Appl. Opt. 39, 2719-2726 (2000). 